\title{
Converse growth estimates for ODEs with slowly growing solutions
}

\author{
Janne Gröhn ${ }^{1}$
}

Received: 4 December 2019 / Accepted: 26 July 2020 / Published online: 17 September 2020

(c) The Author(s) 2020

\begin{abstract}
Let $f_{1}, f_{2}$ be linearly independent solutions of $f^{\prime \prime}+A f=0$, where the coefficient $A$ is an analytic function in the open unit disc $\mathbb{D}$ of the complex plane $\mathbb{C}$. It is shown that many properties of this differential equation can be described in terms of the subharmonic auxiliary function $u=-\log \left(f_{1} / f_{2}\right)^{\#}$. For example, the case when $\sup _{z \in \mathbb{D}}|A(z)|\left(1-|z|^{2}\right)^{2}<\infty$ and $f_{1} / f_{2}$ is normal, is characterized by the condition $\sup _{z \in \mathbb{D}}|\nabla u(z)|\left(1-|z|^{2}\right)<\infty$. Different types of Blaschke-oscillatory equations are also described in terms of harmonic majorants of $u$. Even if $f_{1}, f_{2}$ are bounded linearly independent solutions of $f^{\prime \prime}+A f=0$, it is possible that $\sup _{z \in \mathbb{D}}|A(z)|\left(1-|z|^{2}\right)^{2}=\infty$ or $f_{1} / f_{2}$ is non-normal. These results relate to sharpness discussion of recent results in the literature, and are succeeded by a detailed analysis of differential equations with bounded solutions. Analogues for the Nevanlinna class are also considered, by taking advantage of Nevanlinna interpolating sequences. It is shown that, instead of considering solutions with prescribed zeros, it is possible to construct a bounded solution of $f^{\prime \prime}+A f=0$ in such a way that it solves an interpolation problem natural to bounded analytic functions, while $|A(z)|^{2}\left(1-|z|^{2}\right)^{3} d m(z)$ remains to be a Carleson measure.
\end{abstract}

Keywords Blaschke product · Bounded solution - Growth of solution · Interpolation · Linear differential equation · Nevanlinna class · Normal function · Oscillation of solution

Mathematics Subject Classification Primary 34C11; Secondary 34C10

\section{Introduction}

Let $\operatorname{Hol}(\mathbb{D})$ be the collection of analytic functions in the open unit disc $\mathbb{D}$ of the complex plane $\mathbb{C}$. For $0 \leq \alpha<\infty$, let $L_{\alpha}^{\infty}$ denote the space of $f: \mathbb{D} \rightarrow \mathbb{C}$ for which $\|f\|_{L_{\alpha}^{\infty}}=$ $\sup _{z \in \mathbb{D}}|f(z)|\left(1-|z|^{2}\right)^{\alpha}<\infty$, and write $H_{\alpha}^{\infty}=L_{\alpha}^{\infty} \cap \operatorname{Hol}(\mathbb{D})$ and $H^{\infty}=H_{0}^{\infty}$ for short. We are interested in the relation between the growth of the coefficient $A \in \operatorname{Hol}(\mathbb{D})$ and the

The author was supported in part by the Academy of Finland \#286877.

Janne Gröhn

janne.grohn@uef.fi

1 Department of Physics and Mathematics, University of Eastern Finland, P.O. Box 111, FI-80101

Joensuu, Finland 
oscillation and growth of solutions of

$$
f^{\prime \prime}+A f=0 .
$$

By [43, Theorems 3-4], the following conditions are equivalent:

(i) $A \in H_{2}^{\infty}$;

(ii) zero-sequences of all non-trivial solutions $(f \not \equiv 0)$ of $(1)$ are separated with respect to the hyperbolic metric.

We refer to [3] for a far reaching generalization concerning the connection between the growth of the coefficient $A \in \operatorname{Hol}(\mathbb{D})$ and the minimal separation of zeros of non-trivial solutions of (1). It has been unclear whether

(iii) all solutions of (1) belong to the Korenblum space $\bigcup_{0<\alpha<\infty} H_{\alpha}^{\infty}$,

is equivalent to the conditions above. Recall that, if $f_{1}, f_{2}$ are linearly independent solutions of (1) for $A \in \operatorname{Hol}(\mathbb{D})$, then the Wronskian determinant $W\left(f_{1}, f_{2}\right)=f_{1} f_{2}^{\prime}-f_{1}^{\prime} f_{2}$ reduces to a non-zero complex constant, and consequently, any solution of (1) can be written as a linear combination of $f_{1}, f_{2}$.

In view of results in the literature, the condition (iii) is a natural candidate for a description of the growth of solutions of (1) under (i). Pommerenke used a classical comparison theorem [40, Example 1] to prove that (i) $\Rightarrow$ (iii). This implication has been rediscovered with different methods: growth estimates [20, Theorem 4.3(2)], [23, Theorem 3.1]; successive approximations [9, Theorem I]; and straight-forward integration [16, Theorem 2], [24, Corollary 4(a)]. We point out that, even if $\|A\|_{H_{2}^{\infty}}>0$ is arbitrarily small, some solutions of (1) may be unbounded. Any coefficient condition $A \in H_{\alpha}^{\infty}$ for $0<\alpha<2$ implies boundedness of all solutions of (1) by [20, Theorem 4.3(1)]. For more involved growth estimates in the case of slowly growing solutions, see $[12,14]$.

The difficulty in the converse assertion (iii) $\Rightarrow$ (i) lies in the fact that the assumption concerns all solutions. The existence of one non-trivial slowly growing solution is not sufficient, as $f(z)=\exp (-(1+z) /(1-z))$ is a bounded solution of $(1)$ for $A(z)=-4 z /(1-z)^{4}$, $z \in \mathbb{D}$. Two classical methods to attack problems of this type are the Bank-Laine approach and arguments based on the Schwarzian derivative. In the former case, let $E=f_{1} f_{2}$ denote the product of two linearly independent solutions of (1) for $A \in \operatorname{Hol}(\mathbb{D})$. By [27, pp. 76-77],

$$
4 A=\left(\frac{E^{\prime}}{E}\right)^{2}-\left(\frac{W\left(f_{1}, f_{2}\right)}{E}\right)^{2}-2 \frac{E^{\prime \prime}}{E} .
$$

The Bank-Laine representation is usually used in conjunction with estimates that appear in Wiman-Valiron and Nevanlinna theories. The latter method is based on [27, Theorem 6.1]: if $f_{1}, f_{2}$ are linearly independent solutions of (1) for $A \in \operatorname{Hol}(\mathbb{D})$, then $w=f_{1} / f_{2}$ is a locally univalent meromorphic function in $\mathbb{D}$ such that the Schwarzian derivative

$$
S_{w}=\left(\frac{w^{\prime \prime}}{w^{\prime}}\right)^{\prime}-\frac{1}{2}\left(\frac{w^{\prime \prime}}{w^{\prime}}\right)^{2}
$$

is not only analytic in $\mathbb{D}$ but also satisfies $S_{w}=2 A$. Both approaches represent the coefficient function $A$ in terms of the linearly independent solutions $f_{1}, f_{2}$, and are indispensable tools in the case of fast growing solutions (and also in oscillation theory). However, if all solutions are slowly growing functions in $\mathbb{D}$, then neither of these techniques seem to be sufficiently delicate to produce sharp growth estimates for the coefficient $A$. 


\section{Results}

Many of the following results are converse growth estimates as they measure the growth of the coefficient in terms of solutions. We begin with studying equations with bounded solutions. The preliminary results in Sect. 2.1 not only set the stage for forthcoming findings but also provide a sharpness discussion for [12,44]. The significant part of this article is devoted to the study of the subharmonic auxiliary function $u=-\log \left(f_{1} / f_{2}\right)^{\#}$ where $f_{1}, f_{2}$ are linearly independent solutions of (1). This approach leads to several new characterizations which are, in essence, based on identities obtained in Sect. 2.2. Our intention is to compare properties of $u$ to the coefficient $A$, to the quotient $f_{1} / f_{2}$ and to any non-trivial solution of (1). Results concerning equations with bounded solutions have natural counterparts in the setting of the Nevanlinna class, which are considered in Sect. 2.4. These results depend on recent advances concerning Nevanlinna interpolating sequences. Finally, in Sect. 2.6, we show that fixed points can be prescribed for a solution of (1) in such a way that all solutions remain bounded.

\subsection{Bounded solutions}

The following result indicates that the implication (iii) $\Rightarrow$ (i), mentioned in the Introduction, fails to be true.

\section{Theorem 1 Consider the differential equation (1) in $\mathbb{D}$.}

(i) There exists $A \in \operatorname{Hol}(\mathbb{D}) \backslash H_{2}^{\infty}$ such that all solutions of (1) are bounded.

(ii) Let $0<p<\infty$. There exists $A \in \operatorname{Hol}(\mathbb{D}) \backslash H_{2}^{\infty}$ such that all solutions of (1) belong to $H_{p}^{\infty}$ while one of the solutions is non-normal.

The class of normal functions consists of those meromorphic functions for which $\sup _{z \in \mathbb{D}} w^{\#}(z)\left(1-|z|^{2}\right)<\infty$, where $w^{\#}=\left|w^{\prime}\right| /\left(1+|w|^{2}\right)$ is the spherical derivative. A function $w$ meromorphic in $\mathbb{D}$ is normal if and only if

$$
\{w \circ \varphi: \varphi \text { conformal automorphism of } \mathbb{D}\}
$$

is a normal family in $\mathbb{D}$ in the sense of Montel [30]. We consider the normality of solutions of (1) as well as the normality of the quotient of two linearly independent solutions. If $A \in H_{2}^{\infty}$, then normal solutions of (1) are described by [17, Proposition 7], and the case when the quotient is normal will be characterized in Sect. 2.5. Note that the coefficient condition $A \in H_{2}^{\infty}$ allows non-normal solutions by [10, Theorem 3] and [11, Theorem 1]; and even the normality of all solutions is not sufficient for $A \in H_{2}^{\infty}$ by Theorem 1(i) above.

If $f_{1}, f_{2} \in H^{\infty}$ are linearly independent solutions of (1) for $A \in \operatorname{Hol}(\mathbb{D})$, then $A \in H_{3}^{\infty}$ by a result of Steinmetz [44, p. 130]. Theorem 1(i) shows that this result cannot be improved to $A \in H_{2}^{\infty}$. The intermediate conclusion $A \in H_{\alpha}^{\infty}$ for $\alpha=5 / 2$ has been obtained in [12, Theorem 6] under the weaker assumption $f_{1}, f_{2} \in \mathcal{B}$, while the question of finding the best possible $\alpha$ remains open. Here $\mathcal{B}$ is the Bloch space, which contains $f \in \operatorname{Hol}(\mathbb{D})$ for which $\|f\|_{\mathcal{B}}=\left\|f^{\prime}\right\|_{H_{1}^{\infty}}<\infty$. The desired conclusion $A \in H_{2}^{\infty}$ has been obtained in [12, Theorem 7] under the additional assumption $\inf _{z \in \mathbb{D}}\left(\left|f_{1}(z)\right|+\left|f_{2}(z)\right|\right)>0$. We proceed to state two generalizations in this respect. Theorem 15 in Sect. 4 shows that it is not necessary to take the infimum over the whole unit disc while Theorem 2 below implies that we may take the infimum of a function which is significantly larger than $\left|f_{1}\right|+\left|f_{2}\right|$. The latter generalization is based on having specific information about the structure of the ideal $I_{H^{\infty}}\left(f_{1}, f_{2}\right)$ generated by the solutions $f_{1}, f_{2} \in H^{\infty}$. 
A positive Borel measure $\mu$ on $\mathbb{D}$ is called a Carleson measure, if for fixed $0<p<\infty$ there exists $C=C(p)$ with $0<C<\infty$ such that

$$
\int_{\mathbb{D}}|f(z)|^{p} d \mu(z) \leq C \lim _{r \rightarrow 1^{-}} \frac{1}{2 \pi} \int_{0}^{2 \pi}\left|f\left(r e^{i \theta}\right)\right|^{p} d \theta=C\|f\|_{H^{p}}^{p}, \quad f \in \operatorname{Hol}(\mathbb{D}) .
$$

Here $H^{p}$ is the standard Hardy space. By [8, Lemma 3.3, p. 231], such measures $\mu$ are characterized by $\sup _{a \in \mathbb{D}} \int_{\mathbb{D}}\left|\varphi_{a}^{\prime}(z)\right| d \mu(z)<\infty$, where $\varphi_{a}(z)=(\zeta-z) /(1-\bar{a} z)$ is a conformal automorphism of $\mathbb{D}$ which coincides with its own inverse. Since $|A|^{2}$ is subharmonic for $A \in \operatorname{Hol}(\mathbb{D})$, we deduce $A \in H_{2}^{\infty}$ whenever $|A(z)|^{2}\left(1-|z|^{2}\right)^{3} d m(z)$ is a Carleson measure. This Carleson measure condition appears several times in the literature: in connection to solutions of (1) with uniformly separated zeros [11,15] and in relation to solutions in Hardy spaces $[14,17]$.

Theorem 2 If $f_{1}, f_{2} \in H^{\infty}$ are linearly independent solutions of (1) for $A \in \operatorname{Hol}(\mathbb{D})$ such that

$$
\inf _{a \in \mathbb{D}} \sum_{k=0}^{n}\left(\left|\left(f_{1} \circ \varphi_{a}\right)^{(k)}(0)\right|+\left|\left(f_{2} \circ \varphi_{a}\right)^{(k)}(0)\right|\right)>0
$$

for some $n \in \mathbb{N} \cup\{0\}$, then $|A(z)|^{2}\left(1-|z|^{2}\right)^{3} d m(z)$ is a Carleson measure.

Let $f_{1}, f_{2} \in H^{\infty}$ be linearly independent solutions of (1) for $A \in \operatorname{Hol}(\mathbb{D})$. In [44], Steinmetz proved $\left(f_{1} / f_{2}\right)^{\#} \in L_{2}^{\infty}$ and asked whether this can be improved to $\left(f_{1} / f_{2}\right)^{\#} \in$ $L_{1}^{\infty}$ ? It turns out that Steinmetz's result is best possible up to a multiplicative constant. Recall that the sequence $\left\{z_{n}\right\} \subset \mathbb{D}$ is said to be uniformly separated, if it is separated in the hyperbolic metric and $\sum_{n}\left(1-\left|z_{n}\right|\right) \delta_{z_{n}}$ is a Carleson measure. Here $\delta_{z_{n}}$ is the Dirac measure with point mass at $z_{n} \in \mathbb{D}$.

Theorem 3 Let $\Lambda \subset \mathbb{D}$ be uniformly separated. Then, there exists $A \in \operatorname{Hol}(\mathbb{D})$ such that $|A(z)|^{2}\left(1-|z|^{2}\right)^{3} d m(z)$ is a Carleson measure and (1) admits two linearly independent solutions $f_{1}, f_{2} \in H^{\infty}$ such that $\inf _{z_{n} \in \Lambda}\left(f_{1} / f_{2}\right)^{\#}\left(z_{n}\right)\left(1-\left|z_{n}\right|^{2}\right)^{2}>0$.

In [7, Theorem 1.1], Fournier, Kraus and Roth obtain sharp estimates for $w^{\#}(0)$, where $w$ is a meromorphic function in $\mathbb{D}$ with spherical derivative uniformly bounded away from zero.

Instead of considering prescribed zeros of solutions-which is the approach in Theorem 3 , among many other results-we may also consider solutions which satisfy an interpolation problem natural for bounded analytic functions. Such result has been the objective of recent research. Our solution to this problem is based on combining classical interpolation results by Earl and Øyma.

Theorem 4 Let $\left\{z_{n}\right\} \subset \mathbb{D}$ be uniformly separated and $\left\{w_{n}\right\} \subset \mathbb{C}$ bounded. Then, there exists $A \in \operatorname{Hol}(\mathbb{D})$ such that $|A(z)|^{2}\left(1-|z|^{2}\right)^{3} d m(z)$ is a Carleson measure (1) admits a solution $f \in H^{\infty}$ which satisfies $f\left(z_{n}\right)=w_{n}$ for all $n$, while all solutions of (1) are bounded.

In Sect. 5 we consider oscillation of solutions of such differential equations whose solutions are bounded, and concentrate on the zeros and critical points. 


\subsection{Identities}

We take a short side-track to consider properties of the differential equation (1) assuming that the coefficient $A$ is merely analytic in $\mathbb{D}$. Suppose for a moment that $f$ is a zero-free solution of (1). In this case $\log f \in \operatorname{Hol}(\mathbb{D})$ and

$$
A=-f^{\prime \prime} / f=-(\log f)^{\prime \prime}-\left((\log f)^{\prime}\right)^{2} .
$$

Our next objective is to obtain a similar representation which takes account on both linearly independent solutions and allows them to have zeros in $\mathbb{D}$. Let

$$
\partial f=\frac{1}{2}\left(\frac{\partial f}{\partial x}-i \frac{\partial f}{\partial y}\right), \quad \bar{\partial} f=\frac{1}{2}\left(\frac{\partial f}{\partial x}+i \frac{\partial f}{\partial y}\right),
$$

denote the complex partial derivatives of $f$. Note that $\partial f$ and $\bar{\partial} f$ exist as long as $\partial f / \partial x$ and $\partial f / \partial y$ exist, and then the gradient $\nabla f=(\partial f / \partial x, \partial f / \partial y)$ satisfies $|\nabla f|^{2}=2\left(|\partial f|^{2}+\right.$ $|\bar{\partial} f|^{2}$ ). If $f$ has continuous second-order derivatives (denoted by $f \in C^{2}$ ), then the Laplacian $\Delta f$ can be written in the form $\Delta f=4 \bar{\partial} \partial f=4 \partial \bar{\partial} f$.

We have been unable to find a reference for the following result, which is known for experts in another form. We will present a short proof of Theorem 5 for the convenience of the reader.

Theorem 5 Let $f_{1}, f_{2}$ be linearly independent solutions of (1) for $A \in \operatorname{Hol}(\mathbb{D})$, and define $u=-\log \left(f_{1} / f_{2}\right)^{\#}$. Then,

(i) $\Delta u=4 e^{-2 u}$;

(ii) $\Delta u+|\nabla u|^{2}=e^{-u} \Delta e^{u}$;

(iii) $A=-\partial^{2} u-(\partial u)^{2}$.

Let $f_{1}, f_{2}$ be linearly independent solutions of (1) for $A \in \operatorname{Hol}(\mathbb{D})$. The function $u=$ $-\log \left(f_{1} / f_{2}\right)^{\#}$ has several interesting properties, which make up the bulk of this paper. The underlying reason for the relevance of $u$ is its connection to regular conformal metrics of constant curvature. Actually, $u$ is closely related to the general solution of Liouville's equation in the case of $\mathbb{D}$. This point of view is elaborated further in Remark 1, Sect. 6. For the classical representation of regular conformal metrics of constant curvature in terms of analytic functions, see [31]. Nevertheless, we choose to proceed without the notation of conformal metrics.

Theorem 5(i) implies $\Delta u=4\left(\left(f_{1} / f_{2}\right)^{\#}\right)^{2} \geq 0$. Therefore $u$ is subharmonic, and $r \mapsto(1 /(2 \pi)) \int_{0}^{2 \pi} u\left(r e^{i \theta}\right) d \theta$ is a non-decreasing and convex function of $\log r$. Theorem 5(iii) is a counterpart of (3). As $W\left(f_{1}, f_{2}\right)$ is a non-zero complex constant, $\partial u=$ $\left(f_{1}^{\prime} \bar{f}_{1}+f_{2}^{\prime} \bar{f}_{2}\right) /\left(\left|f_{1}\right|^{2}+\left|f_{2}\right|^{2}\right)$ is finite-valued throughout $\mathbb{D}$.

\subsection{Blaschke-oscillatory equations}

The differential equation (1) is said to be Blaschke-oscillatory, if $A \in \operatorname{Hol}(\mathbb{D})$ and the zerosequence $\left\{z_{n}\right\}$ of any non-trivial solution of (1) satisfies the Blaschke condition $\sum_{n}(1-$ $\left.\left|z_{n}\right|\right)<\infty$. Such differential equations are characterized by the fact that the quotient of any two linearly independent solutions belongs to the Nevanlinna class [22, Lemma 3]. The Nevanlinna class $\mathcal{N}$ consists of those meromorphic functions $w$ in $\mathbb{D}$ such that $\int_{\mathbb{D}} w^{\#}(z)^{2}(1-$ $\left.|z|^{2}\right) d m(z)<\infty$; see Sect. 7. A meromorphic function $w$ is said to be of uniformly bounded 
characteristic, that is $w \in \mathrm{UBC}$, if $w^{\#}(z)^{2}\left(1-|z|^{2}\right) d m(z)$ is a Carleson measure. We refer to [38, Theorem 3] for more details.

Let $u \neq \equiv-\infty$ be a subharmonic function in $\mathbb{D}$. A harmonic function $h$ is said to be a harmonic majorant for $u$ if $u \leq h$ in $\mathbb{D}$. The least harmonic majorant $\hat{u}$ is a harmonic majorant which is point-wise smaller than any other harmonic majorant for $u$. If $f \in \operatorname{Hol}(\mathbb{D})$, then it is well-known that $f \in \mathcal{N}$ if and only if $\log ^{+}|f|$ admits a harmonic majorant, while $f \in H^{p}$ if and only if $|f|^{p}$ has a harmonic majorant.

Theorem 6 Let $f_{1}, f_{2}$ be linearly independent solutions of (1) for $A \in \operatorname{Hol}(\mathbb{D})$, and define $u=-\log \left(f_{1} / f_{2}\right)^{\#}$. Then,

(i) $f_{1} / f_{2} \in \mathcal{N}$ if and only if $u$ has a harmonic majorant;

(ii) $f_{1} / f_{2} \in \mathcal{N}$ and is normal if and only if $u_{a}(z)=u(a+(1-|a|) z)-u(a), a \in \mathbb{D}$, have harmonic majorants with $\sup _{a \in \mathbb{D}} \widehat{u_{a}}(0)<\infty$;

(iii) $f_{1} / f_{2} \in \mathrm{UBC}$ if and only if $\sup _{a \in \mathbb{D}}(\hat{u}(a)-u(a))<\infty$.

\section{Moreover,}

(iv) all solutions of (1) belong to $\mathcal{N}$ if and only if $u$ has a positive harmonic majorant;

(v) all solutions of (1) belong to $H^{p}$, for $0<p<\infty$, if and only if $\exp \left(\frac{p}{2} u\right)$ has a harmonic majorant;

(vi) all solutions of (1) belong to $H^{\infty}$ if and only if $\exp (u) \in L^{\infty}$.

Recall that the following conditions are equivalent for any subharmonic function $u$ in the unit disc (see [8, p. 66] for more details): (a) $u$ has a positive harmonic majorant; (b) the subharmonic function $u^{+}=\max \{u, 0\}$ has a harmonic majorant; (c) $u$ is majorized by a Poisson integral of a finite measure on $\partial \mathbb{D}$. In Theorem 6 , it is possible that $u$ admits a harmonic majorant which takes negative values, since there are Blaschke-oscillatory equations (1) whose non-trivial solutions lie outside $\mathcal{N}$ [22, Section 4.3]. Although the items (iv)-(vi) are immediate, their assertions raise an interesting observation. Since we may describe the behavior of all solutions of (1) in terms of $f_{1} / f_{2}$, no essential information is reduced in this quotient. In Remark 2, Sect. 7, we illustrate that the growth of solutions of Blaschke-oscillatory equations is severely restricted.

There are normal functions which do not belong to $\mathcal{N}$. Classical example of such a function is the elliptic modular function [30, p. 57]. If $f_{1}, f_{2}$ are linearly independent solutions of (1) for $A \in \operatorname{Hol}(\mathbb{D})$, then $f_{1} / f_{2} \in \mathcal{N}$ provided that $f_{1} / f_{2}$ is normal and the set where $\left|f_{1}\right|^{2}+\left|f_{2}\right|^{2}$ takes small values, is not too large.

Proposition 1 Let $f_{1}, f_{2}$ be linearly independent solutions of (1) for $A \in \operatorname{Hol}(\mathbb{D})$. The differential equation (1) is Blaschke-oscillatory if $f_{1} / f_{2}$ is normal and there exists $0<\delta<\infty$ such that $\int_{\left\{z \in \mathbb{D}:\left|f_{1}(z)\right|^{2}+\left|f_{2}(z)\right|^{2}<\delta\right\}} d m(z) /\left(1-|z|^{2}\right)<\infty$.

\subsection{Nevanlinna interpolating sequences}

By recent advances concerning free interpolation in $\mathcal{N}[18,19,32]$, there is an astounding resemblance between uniformly separated sequences and Nevanlinna interpolating sequences. Therefore the following results can be interpreted as Nevanlinna analogues of ones that are either presented in Sect. 2.1 or already appear in the literature.

A sequence $\Lambda \subset \mathbb{D}$ is called (free) interpolating for $\mathcal{N}$ if the trace of $\mathcal{N}$ on $\Lambda$ is ideal [18, p. 3]. That is, for any $g \in \mathcal{N}$ and for any bounded sequence $\left\{w_{n}\right\} \in \mathbb{C}$, there exists $f \in \mathcal{N}$ such that $f\left(z_{n}\right)=w_{n} g\left(z_{n}\right)$ for all $z_{n} \in \Lambda$. The collection of (free) interpolating sequences 
for $\mathcal{N}$ is denoted by Int $\mathcal{N}$. Note that $\Lambda \in \operatorname{Int} \mathcal{N}$ if and only if the trace $\mathcal{N} \mid \Lambda$ contains all bounded sequences [18, Remark 1.1], and in particular, all sequences in Int $\mathcal{N}$ satisfy the Blaschke condition.

Let $\operatorname{Har}^{+}(\mathbb{D})$ denote the space of positive harmonic functions in $\mathbb{D}$. By [18, Theorem 1.2], $\Lambda \in \operatorname{Int} \mathcal{N}$ if and only if there exists $h \in \operatorname{Har}^{+}(\mathbb{D})$ such that

$$
\prod_{z_{k} \in \Lambda \backslash\left\{z_{n}\right\}}\left|\frac{z_{k}-z_{n}}{1-\bar{z}_{k} z_{n}}\right| \geq e^{-h\left(z_{n}\right)}, \quad z_{n} \in \Lambda .
$$

The reader is invited to compare (4) to the classical description (20) of uniformly separated sequences, which are precisely the interpolating sequences for $H^{\infty}$.

Theorem 7 Let $\Lambda \in \operatorname{Int} \mathcal{N}$. Then, there exist $h \in \operatorname{Har}^{+}(\mathbb{D})$ and $A \in \operatorname{Hol}(\mathbb{D})$ such that $|A(z)|\left(1-|z|^{2}\right)^{2} \leq e^{h(z)}, z \in \mathbb{D}$, and (1) admits a non-trivial solution whose zero-sequence is $\Lambda$.

By [18, Corollary 1.9], Theorem 7 allows us the prescribe any separated Blaschke sequence to be a zero-sequence of a non-trivial solution of (1). Theorem 7 should be compared to [11, Theorem 1], according to which any separated sequence of sufficiently small upper uniform density can appear as a subset of the zero-sequence of a non-trivial solution of (1) under the coefficient condition $A \in H_{2}^{\infty}$. The coefficient condition in Theorem 7 is of different nature as it controls the growth in an average sense. On one hand, the restriction $|A(z)|\left(1-|z|^{2}\right)^{2} \leq e^{h(z)}, z \in \mathbb{D}$ and $h \in \operatorname{Har}^{+}(\mathbb{D})$, passes through functions such as $A(z)=(e /(1-z))^{k}$ for any $0<k<\infty$. On the other hand, it implies that there exists $0<C<\infty$ such that

$$
\int_{0}^{2 \pi} \log ^{+}\left|A\left(r e^{i \theta}\right)\right| d \theta \leq 2 \log ^{+} \frac{1}{1-r}+C, \quad r \rightarrow 1^{-},
$$

which is an estimate that cannot be improved even if $A \in H_{2}^{\infty}$. Estimate (5) reveals that such coefficient $A$ lies close to $\mathcal{N}$ as it is non-admissible.

The following result is an analogue of [10, Theorem 5], and is related to the classical 0, 1interpolation result due to Carleson [2, Theorem 2]. The Nevanlinna counterpart of Carleson's result is presented in Sect. 9.

Theorem 8 Assume that $\alpha, \beta \in \mathbb{C} \backslash\{0\}$ are distinct values. Let $\left\{z_{n}\right\},\left\{\zeta_{n}\right\}$ be any Blaschke sequences, and let $B_{\left\{z_{n}\right\}}$ and $B_{\left\{\zeta_{n}\right\}}$ be the corresponding Blaschke products. If there exists $h \in \mathrm{Har}^{+}(\mathbb{D})$ such that

$$
\left|B_{\left\{z_{n}\right\}}(z)\right|+\left|B_{\left\{\zeta_{n}\right\}}(z)\right| \geq e^{-h(z)}, \quad z \in \mathbb{D},
$$

then there exists $A \in \operatorname{Hol}(\mathbb{D})$ and $H \in \operatorname{Har}^{+}(\mathbb{D})$ such that $|A(z)|\left(1-|z|^{2}\right)^{2} \leq e^{H(z)}, z \in \mathbb{D}$, and (1) admits a solution $f$ with $f\left(z_{n}\right)=\alpha$ and $f\left(\zeta_{n}\right)=\beta$ for all $n$.

We turn to study differential equations with solutions in $\mathcal{N}$. It turns out that Steinmetz's approach from [44, Theorem, p. 129] applies with obvious changes.

Theorem 9 If $f_{1}, f_{2} \in \mathcal{N}$ are linearly independent solutions of $(1)$ for $A \in \operatorname{Hol}(\mathbb{D})$, then there exists $H \in \operatorname{Har}^{+}(\mathbb{D})$ such that $|A(z)|\left(1-|z|^{2}\right)^{3} \leq e^{H(z)}$ and $\left(f_{1} / f_{2}\right)^{\#}(z)\left(1-|z|^{2}\right)^{2} \leq e^{H(z)}$, $z \in \mathbb{D}$.

We may also ask when the stronger estimate $|A(z)|\left(1-|z|^{2}\right)^{2} \leq e^{H(z)}, z \in \mathbb{D}$, is obtained? The following result is analogous to Theorem 2; generalization of the assumption (7) to higher derivatives is left to the interested reader. 
Theorem 10 If $f_{1}, f_{2} \in \mathcal{N}$ are linearly independent solutions of (1) for $A \in \operatorname{Hol}(\mathbb{D})$ such that

$$
\sum_{j=1,2}\left(\left|f_{j}(z)\right|+\left|f_{j}^{\prime}(z)\right|\left(1-|z|^{2}\right)\right) \geq e^{-h(z)}, \quad z \in \mathbb{D},
$$

for $h \in \operatorname{Har}^{+}(\mathbb{D})$, then there exists $H \in \operatorname{Har}^{+}(\mathbb{D})$ such that $|A(z)|\left(1-|z|^{2}\right)^{2} \leq e^{H(z)}, z \in \mathbb{D}$.

The sequence $\Lambda \subset \mathbb{D}$ is called $h$-separated, if there exists $h \in \operatorname{Har}^{+}(\mathbb{D})$ such that the pseudo-hyperbolic discs $\Delta_{p}\left(z_{n}, e^{-h\left(z_{n}\right)}\right), z_{n} \in \Lambda$, are pairwise disjoint. Recall that the pseudo-hyperbolic disc of radius $0<\delta<1$, centered at $z \in \mathbb{D}$, is given by $\Delta_{p}(z, \delta)=\left\{w \in \mathbb{D}: \varrho_{p}(z, w)<\delta\right\}$ where $\varrho_{p}(z, w)=|w-z| /|1-\bar{w} z|$ is the pseudohyperbolic distance between $z, w \in \mathbb{D}$. The following result corresponds to Schwarz's findings [43, Theorems 3-4] in the case $A \in H_{2}^{\infty}$.

Proposition 2 Suppose that there exist $A \in \operatorname{Hol}(\mathbb{D})$ and $H \in \operatorname{Har}^{+}(\mathbb{D})$ such that $|A(z)|(1-$ $\left.|z|^{2}\right)^{2} \leq e^{H(z)}, z \in \mathbb{D}$. Then, there exists $h \in \operatorname{Har}^{+}(\mathbb{D})$ such that the zero-sequence of any non-trivial solution of (1) is h-separated.

Conversely, suppose that $A \in \operatorname{Hol}(\mathbb{D})$ and there exists $h \in \mathrm{Har}^{+}(\mathbb{D})$ such that the zerosequence of any non-trivial solution of (1) is h-separated. Then, there exists $H \in \mathrm{Har}^{+}(\mathbb{D})$ such that $|A(z)|\left(1-|z|^{2}\right)^{2} \leq e^{H(z)}, z \in \mathbb{D}$.

\subsection{Point-wise growth restrictions}

A function $\omega: \mathbb{D} \rightarrow(0, \infty)$ is said to be a weight if it is bounded and continuous. The weight $\omega$ is radial if $\omega(z)=\omega(|z|)$ for all $z \in \mathbb{D}$, and is called regular if it is radial and for each $0 \leq s<1$ there exists a constant $C=C(s, \omega)$ with $1 \leq C<\infty$ such that

$$
C^{-1} \omega(t) \leq \omega(r) \leq C \omega(t), \quad 0 \leq r \leq t \leq r+s(1-r)<1 .
$$

For a general reference for regular weights, see [39, Chapter 1]. For a weight $\omega$, let $L_{\omega}^{\infty}$ denote the growth space which consists of functions $f: \mathbb{D} \rightarrow \mathbb{C}$ for which $\|f\|_{L_{\omega}^{\infty}}=$ $\sup _{z \in \mathbb{D}}|f(z)| \omega(z)<\infty$, and denote $H_{\omega}^{\infty}=L_{\omega}^{\infty} \cap \operatorname{Hol}(\mathbb{D})$.

Theorem 11 Let $f_{1}, f_{2}$ be linearly independent solutions of $(1)$ for $A \in \operatorname{Hol}(\mathbb{D})$, and define $u=-\log \left(f_{1} / f_{2}\right)^{\#}$. Suppose that $\omega$ is a regular weight which satisfies $\sup _{z \in \mathbb{D}} \omega(z) /(1-$ $|z|)<\infty$. Then, $|\nabla u| \in L_{\omega}^{\infty}$ if and only if $A \in H_{\omega^{2}}^{\infty}$ and $\left(f_{1} / f_{2}\right)^{\#} \in L_{\omega}^{\infty}$.

The following result follows directly from Theorem 11 with $\omega(z)=1-|z|^{2}, z \in \mathbb{D}$. This corollary concerns those differential equations (1) which have both desired properties mentioned in Sect. 2.1: $A \in H_{2}^{\infty}$ and $\left(f_{1} / f_{2}\right)^{\#} \in L_{1}^{\infty}$.

Corollary 1 Let $f_{1}, f_{2}$ be linearly independent solutions of $(1)$ for $A \in \operatorname{Hol}(\mathbb{D})$, and define $u=-\log \left(f_{1} / f_{2}\right)^{\#}$. Then, $|\nabla u| \in L_{1}^{\infty}$ if and only if $A \in H_{2}^{\infty}$ and $f_{1} / f_{2}$ is normal.

Corollary 1 can also be deduced by combining several results in the literature. The first part follows from [1, Theorem 6], while the second part can be concluded from [48, Theorem 1] and [48, Corollary to Theorem 2]. Note that $f_{1} / f_{2}$ is uniformly locally univalent provided that $A \in H_{2}^{\infty}$, which can be seen by applying Nehari's univalency criterion [34, Theorem I] locally. 
Corollary 2 Let $f_{1}, f_{2}$ be linearly independent solutions of (1) for $A \in H_{\omega^{2}}^{\infty}$, and define $u=-\log \left(f_{1} / f_{2}\right)^{\#}$. Suppose that $\omega$ is a regular weight which satisfies $\sup _{z \in \mathbb{D}} \omega(z) /(1-$ $|z|)<\infty$. Then, the following statements are equivalent:

(i) $|\nabla u| \in L_{\omega}^{\infty}$;

(ii) $\left(f_{1} / f_{2}\right)^{\#} \in L_{\omega}^{\infty}$;

(iii) $\left(\left|f_{1}^{\prime}\right|+\left|f_{2}^{\prime}\right|\right) /\left(\left|f_{1}\right|+\left|f_{2}\right|\right) \in L_{\omega}^{\infty}$;

(iv) $\Delta u \in L_{\omega^{2}}^{\infty}$.

If $\omega(z)=1-|z|^{2}, z \in \mathbb{D}$, then Corollary 2 provides a complete description of those differential equations (1) for $A \in H_{2}^{\infty}$, where the quotient of two linearly independent solutions is normal. Such characterizations are important in oscillation theory. Since normal functions are Lipschitz-continuous, as mappings from $\mathbb{D}$ equipped with the hyperbolic metric to the Riemann sphere equipped with the chordal metric, the normality of $f_{1} / f_{2}$ implies that its the zeros and poles (which correspond to the zeros of $f_{1}$ and $f_{2}$, respectively) are separated in the hyperbolic metric. Finally, we point out that Corollary 2(iii) does not extend to higher derivatives, since there are differential equations (1) with $A \in \operatorname{Hol}(\mathbb{D})$ and $|A|=$ $\left(\left|f_{1}^{\prime \prime}\right|+\left|f_{2}^{\prime \prime}\right|\right) /\left(\left|f_{1}\right|+\left|f_{2}\right|\right) \in L_{2}^{\infty}$ such that the quotient $f_{1} / f_{2}$ of linearly independent solutions $f_{1}, f_{2}$ is non-normal; see [28] and Theorem 3 .

\subsection{Prescribed fixed points}

The point $z_{0} \in \mathbb{D}$ is said to be a fixed point of $f \in \operatorname{Hol}(\mathbb{D})$ if $f\left(z_{0}\right)=z_{0}$. There are a lot of known results according to which zeros and critical points (i.e., zeros of the derivative) can be prescribed for solutions of (1) for $A \in \operatorname{Hol}(\mathbb{D})$. See [11,13,21,22] among many others. For example, the proof of Theorem 3 depends on such an argument. It turns out that fixed points can be prescribed for a solution of (1) under the coefficient condition $A \in \operatorname{Hol}(\mathbb{D})$ in such a way that all solutions of the differential equation remain bounded. Such differential equations were studied in detail in Sect. 2.1.

Theorem 12 Let $\Lambda \subset \mathbb{D}$ be a Blaschke sequence, and let $0<\varepsilon<1$. Then, there exists a coefficient $A \in \operatorname{Hol}(\mathbb{D})$ such that $|A(z)|^{2}\left(1-|z|^{2}\right)^{3} d m(z)$ is a Carleson measure; the differential equation (1) admits a solution $f$, which satisfies $\|f\|_{H^{\infty}}<1+\varepsilon$ and has fixed points $\{0\} \cup \Lambda$; all solutions of (1) are bounded.

If we assume that prescribed fixed points are uniformly separated, then we can go further and dictate their type. In this paper, we make distinction between three different types: the fixed point $z_{0} \in \mathbb{D}$ of $f \in \operatorname{Hol}(\mathbb{D})$ is said to be attractive if $\left|f^{\prime}\left(z_{0}\right)\right|<1$, neutral if $\left|f^{\prime}\left(z_{0}\right)\right|=1$, and repulsive if $\left|f^{\prime}\left(z_{0}\right)\right|>1$.

Theorem 13 Let $\Lambda \subset \mathbb{D} \backslash\{0\}$ be uniformly separated. Then, there exists a coefficient $A \in$ $\operatorname{Hol}(\mathbb{D})$ such that $|A(z)|^{2}\left(1-|z|^{2}\right)^{3} d m(z)$ is a Carleson measure; the differential equation (1) admits a bounded solution for which every point in $\Lambda$ is a fixed point of prescribed type; all solutions of (1) are bounded.

Theorem 13 has a natural counterpart in the setting of Nevanlinna interpolating sequences. Note that Theorem 12 is valid for sequences $\Lambda \in \operatorname{Int} \mathcal{N}$ as it is.

Theorem 14 Let $\Lambda \subset \mathbb{D} \backslash\{0\}$ and $\Lambda \in \operatorname{Int} \mathcal{N}$. Then, there exists a coefficient $A \in \operatorname{Hol}(\mathbb{D})$ and $H \in \operatorname{Har}^{+}(\mathbb{D})$ such that $|A(z)|^{2}\left(1-|z|^{2}\right)^{2} \leq e^{H(z)}, z \in \mathbb{D}$, and (1) admits a solution for which every point in $\Lambda$ is a fixed point of prescribed type. 


\section{Proof of Theorem 1}

The following argument is based on concrete construction.

Proof (of Theorem 1) (i) Let $0<p<1 / 2$, and

$$
f_{1}(z)=\exp \left(i \cdot \frac{p}{2 \pi}\left(\log \frac{2 i}{1-z}\right)^{2}\right), \quad z \in \mathbb{D} .
$$

Note that the function $z \mapsto 2 i /(1-z)$ maps $\mathbb{D}$ onto $\{z \in \mathbb{C}: \operatorname{Im} z>1\}$. Since

$$
\operatorname{Re}\left(i \cdot \frac{p}{2 \pi}\left(\log \frac{2 i}{1-z}\right)^{2}\right)=-\frac{p}{\pi} \log \frac{2}{|1-z|} \arg \frac{2 i}{1-z}, \quad z \in \mathbb{D},
$$

we deduce $2^{-p}(1-|z|)^{p} \leq\left|f_{1}(z)\right| \leq 1$ for $z \in \mathbb{D}$. Since $f_{1}$ is zero-free, we conclude $A=-f_{1}^{\prime \prime} / f_{1} \in \operatorname{Hol}(\mathbb{D})$. Moreover, $A \notin H_{2}^{\infty}$ because

$$
A(z)=p \frac{p\left(\log \frac{2 i}{1-z}\right)^{2}-i \pi \log \frac{2 i}{1-z}-i \pi}{\pi^{2}(1-z)^{2}}, \quad z \in \mathbb{D} .
$$

It remains to show that all solutions of (1) are bounded. Note that

$$
f_{2}(z)=f_{1}(z) \int_{0}^{z} \frac{1}{f_{1}(\zeta)^{2}} d \zeta, \quad z \in \mathbb{D}
$$

is a bounded solution of (1), and $f_{2}$ is linearly independent to $f_{1}$. Here we integrate along the straight line segment. This completes the proof of (i), since every solution of (1) is a linear combination of $f_{1}, f_{2}$.

(ii) Let $0<p<1 / 2$, and

$$
f_{1}(z)=\exp \left(i \cdot \frac{p}{\pi}\left(\log \frac{1+z}{1-z}\right)^{2}\right), \quad z \in \mathbb{D} .
$$

Similar function has been utilized in [29, pp. 142-143]. We point out that $f_{1}$ has asymptotic values 0 and $\infty$ at $z=1$, and hence $f_{1}$ is not normal. This fact alone implies that the zero-free function $f_{1}$ cannot be a solution of (1) for $A \in H_{2}^{\infty}$; see [17, Proposition 7]. As in the part (i), we deduce

$$
\left(\frac{1-|z|}{1+|z|}\right)^{p} \leq\left|f_{1}(z)\right| \leq\left(\frac{1+|z|}{1-|z|}\right)^{p}, \quad z \in \mathbb{D} .
$$

Since $f_{1}$ is zero-free, we conclude $A=-f_{1}^{\prime \prime} / f_{1} \in \operatorname{Hol}(\mathbb{D})$. Moreover, $A \notin H_{2}^{\infty}$ as

$$
A(z)=8 p \frac{2 p\left(\log \frac{1+z}{1-z}\right)^{2}-i \pi z \log \frac{1+z}{1-z}-i \pi}{\pi^{2}\left(1-z^{2}\right)^{2}}, \quad z \in \mathbb{D} .
$$

It remains to show that all solutions of (1) belong to $H_{p}^{\infty}$. On one hand, it is clear that $f_{1} \in H_{p}^{\infty}$ by (10). On the other hand, (9) is a solution of (1) which is linearly independent to $f_{1}$. Since $z \mapsto \int_{0}^{z} d \zeta / f_{1}(\zeta)^{2}$ is bounded in $\mathbb{D}$, we have $f_{2} \in H_{p}^{\infty}$. This completes the proof of Theorem 1. 


\section{Proof of Theorem 2}

We offer two different proofs for Theorem 2 . We begin by considering a more general result which implies Theorem 2 as a corollary. The following lemma indicates that any analytic function, which satisfies $H_{\alpha}^{\infty}$-type estimate outside a small exceptional set, actually belongs to $H_{\alpha}^{\infty}$.

Lemma 1 Let $f \in \operatorname{Hol}(\mathbb{D})$ and $0 \leq \alpha<\infty$. Then $f \in H_{\alpha}^{\infty}$, if there exist pairwise disjoint $\operatorname{discs} \Delta_{p}\left(z_{n}, \delta\right), z_{n} \in \mathbb{D}$ and $0<\delta<1$, such that

$$
\sup \left\{|f(z)|\left(1-|z|^{2}\right)^{\alpha}: z \in \mathbb{D} \backslash \bigcup_{n} \Delta_{p}\left(z_{n}, \delta\right)\right\}<\infty .
$$

Proof Let $z \in \Delta_{p}\left(z_{n}, \delta\right)$ for some $n$, and let $S$ be the supremum in (11). By the maximum modulus principle, there exists $\zeta \in \partial \Delta_{p}\left(z_{n}, \delta\right)$ such that $|f(\zeta)|=\max \{|f(\xi)|: \xi \in$ $\left.\overline{\Delta_{p}\left(z_{n}, \delta\right)}\right\}$. By the standard estimates, there exists a constant $C=C(\delta)$ with $0<C<\infty$ such that

$$
|f(z)|\left(1-|z|^{2}\right)^{\alpha} \leq|f(\zeta)|\left(1-|z|^{2}\right)^{\alpha} \leq C^{\alpha}|f(\zeta)|\left(1-|\zeta|^{2}\right)^{\alpha} \leq C^{\alpha} S .
$$

The assertion $f \in H_{\alpha}^{\infty}$ follows.

Recall that the space BMOA consists of those functions in $H^{2}$ whose boundary values have bounded mean oscillation on $\partial \mathbb{D}$, or equivalently, of those functions $f \in \operatorname{Hol}(\mathbb{D})$ for which $\left|f^{\prime}(z)\right|^{2}\left(1-|z|^{2}\right) d m(z)$ is a Carleson measure. We write $\|f\|_{\mathrm{BMOA}}^{2}=\sup _{a \in \mathbb{D}}\left\|f_{a}\right\|_{H^{2}}^{2}$ where $f_{a}(z)=f\left(\varphi_{a}(z)\right)-f(a)$ for $a, z \in \mathbb{D}$.

Theorem 15 If $f_{1}, f_{2} \in \mathcal{B}$ are linearly independent solutions of (1) for $A \in \operatorname{Hol}(\mathbb{D})$, and there exist pairwise disjoint discs $\Delta_{p}\left(z_{n}, \delta\right), z_{n} \in \mathbb{D}$ and $0<\delta<1$, with

$$
\inf \left\{\left|f_{1}(z)\right|+\left|f_{2}(z)\right|: z \in \mathbb{D} \backslash \bigcup_{n} \Delta_{p}\left(z_{n}, \delta\right)\right\}>0,
$$

then $A \in H_{2}^{\infty}$. If $f_{1}, f_{2} \in \mathrm{BMOA}$ and the sequence $\left\{z_{n}\right\} \subset \mathbb{D}$ in (12) is uniformly separated, then $|A(z)|^{2}\left(1-|z|^{2}\right)^{3} d m(z)$ is a Carleson measure.

The first part of Theorem 15 improves [12, Theorem 7] by Example 1(ii) below. When comparing Theorem 15 to Theorem 2 note that in the former result it is not required that $f_{1}, f_{2} \in H^{\infty}$.

Proof (of Theorem 15) Let $f_{1}, f_{2} \in \mathcal{B}$ be linearly independent solutions of (1) and suppose that (12) holds. Denote $\Omega=\bigcup_{n} \Delta_{p}\left(z_{n}, \delta\right)$. Since

$$
|A|=\frac{\left|f_{1}\right|+\left|f_{2}\right|}{\left|f_{1}\right|+\left|f_{2}\right|}|A|=\frac{\left|f_{1}^{\prime \prime}\right|+\left|f_{2}^{\prime \prime}\right|}{\left|f_{1}\right|+\left|f_{2}\right|},
$$

we deduce

$$
\sup _{z \in \mathbb{D} \backslash \Omega}|A(z)|\left(1-|z|^{2}\right)^{2} \leq \frac{\left\|f_{1}^{\prime \prime}\right\|_{H_{2}^{\infty}}+\left\|f_{2}^{\prime \prime}\right\|_{H_{2}^{\infty}}}{\inf _{z \in \mathbb{D} \backslash \Omega}\left(\left|f_{1}(z)\right|+\left|f_{2}(z)\right|\right)} .
$$

Since $A \in \operatorname{Hol}(\mathbb{D})$, we conclude $A \in H_{2}^{\infty}$ by Lemma 1 . This completes the proof of the first part of Theorem 15. 
If $f_{1}, f_{2} \in$ BMOA and $\left\{z_{n}\right\} \subset \mathbb{D}$ in (12) is uniformly separated, then we write

$$
\sup _{a \in \mathbb{D}} \int_{\mathbb{D}}|A(z)|^{2}\left(1-|z|^{2}\right)^{3} \frac{1-|a|^{2}}{|1-\bar{a} z|^{2}} d m(z)=I_{1}+I_{2},
$$

where $I_{1}, I_{2}$ are defined as below. By (13) and [42, Theorem 4.2.1], we deduce

$$
\begin{aligned}
I_{1} & =\sup _{a \in \mathbb{D}} \int_{\mathbb{D} \backslash \Omega}|A(z)|^{2}\left(1-|z|^{2}\right)^{3} \frac{1-|a|^{2}}{|1-\bar{a} z|^{2}} d m(z) \\
& \lesssim \sup _{a \in \mathbb{D}} \int_{\mathbb{D}}\left(\left|f_{1}^{\prime \prime}(z)\right|^{2}+\left|f_{2}^{\prime \prime}(z)\right|^{2}\right)\left(1-|z|^{2}\right)^{3} \frac{1-|a|^{2}}{|1-\bar{a} z|^{2}} d m(z)<\infty .
\end{aligned}
$$

Actually, (14) is bounded above by a constant multiple of $\left\|f_{1}\right\|_{\mathrm{BMOA}}^{2}+\left\|f_{2}\right\|_{\mathrm{BMOA}}^{2}$. Since $A \in H_{2}^{\infty}$ by the first part of the proof, standard estimates yield

$$
\begin{aligned}
I_{2} & =\sup _{a \in \mathbb{D}} \sum_{n} \int_{\Delta_{p}\left(z_{n}, \delta\right)}|A(z)|^{2}\left(1-|z|^{2}\right)^{3} \frac{1-|a|^{2}}{|1-\bar{a} z|^{2}} d m(z) \\
& \lesssim\|A\|_{H_{2}^{\infty}}^{2} \sup _{a \in \mathbb{D}} \sum_{n} \frac{\left(1-|a|^{2}\right)\left(1-\left|z_{n}\right|^{2}\right)}{\left|1-\bar{a} z_{n}\right|^{2}}<\infty .
\end{aligned}
$$

The sum in (15) is finite by the uniform separation of $\left\{z_{n}\right\}$. This completes the proof of Theorem 15.

If $\left\{z_{n}\right\} \subset \mathbb{D}$ is a Blaschke sequence, then the Blaschke product

$$
B(z)=B_{\left\{z_{n}\right\}}(z)=\prod_{n} \frac{\left|z_{n}\right|}{z_{n}} \frac{z_{n}-z}{1-\bar{z}_{n} z}, \quad z \in \mathbb{D},
$$

is a bounded analytic function which vanishes precisely on $\left\{z_{n}\right\}$. Let $f_{1}, f_{2} \in H^{\infty}$. By [45, Theorem 3], the ideal

$$
J_{H^{\infty}}\left(f_{1}, f_{2}\right)=\left\{f \in H^{\infty}: \exists c=c(f)>0 \text { such that }|f| \leq c\left(\left|f_{1}\right|+\left|f_{2}\right|\right)\right\}
$$

contains a Blaschke product whose zeros form a finite union of uniformly separated sequences if and only if (2) holds. If $B$ is such a Blaschke product, then there exists a constant $0<\delta<1$ and a subsequence $\left\{z_{n}^{\prime}\right\}$ of zeros of $B$ such that the discs $\Delta_{p}\left(z_{n}^{\prime}, \delta\right), n \in \mathbb{N}$, are pairwise disjoint and

$$
\inf \left\{|B(z)|: z \in \mathbb{D} \backslash \bigcup_{n} \Delta_{p}\left(z_{n}^{\prime}, \delta\right)\right\}>0 .
$$

This follows from [26, Lemmas 1 and 3]; see also [35, Lemma 1]. Therefore Theorem 15 gives an immediate proof for Theorem 2 . We also present another proof which, in addition, provides a concrete representation for the coefficient $A$.

Proof (of Theorem 2) By (2) and [45, Theorem 3], the ideal $I_{H^{\infty}}\left(f_{1}, f_{2}\right)$ contains a Blaschke product $B$ whose zeros form a finite union of uniformly separated sequences. This is equivalent to the fact that there exist functions $g_{1}, g_{2} \in H^{\infty}$ such that $f_{1} g_{1}+f_{2} g_{2}=B$. Differentiate this identity twice, and then apply (1) to $f_{1}^{\prime \prime}$ and $f_{2}^{\prime \prime}$, to obtain

$$
A=\frac{2\left(f_{1}^{\prime} g_{1}^{\prime}+f_{2}^{\prime} g_{2}^{\prime}\right)+f_{1} g_{1}^{\prime \prime}+f_{2} g_{2}^{\prime \prime}-B^{\prime \prime}}{B} .
$$


As in the proof of Theorem 15 , by taking account on (16), we conclude that $|A(z)|^{2}(1-$ $\left.|z|^{2}\right)^{3} d m(z)$ is a Carleson measure.

One of the objectives in Sect. 2.1 was to generalize a result according to which $A \in$ $H_{2}^{\infty}$ if $f_{1}, f_{2} \in \mathcal{B}$ are linearly independent solutions of (1) for $A \in \operatorname{Hol}(\mathbb{D})$ such that $\inf _{z \in \mathbb{D}}\left(\left|f_{1}(z)\right|+\left|f_{2}(z)\right|\right)>0$. The Cauchy-Schwarz inequality gives

$$
\left|W\left(f_{1}, f_{2}\right)\right|^{2} \leq\left(\left|f_{1}\right|^{2}+\left|f_{2}\right|^{2}\right)\left(\left|f_{1}^{\prime}\right|^{2}+\left|f_{2}^{\prime}\right|^{2}\right) .
$$

Since $f_{1}^{\prime}, f_{2}^{\prime} \in H_{1}^{\infty}$, we deduce $\left|f_{1}(z)\right|+\left|f_{2}(z)\right| \gtrsim 1-|z|^{2}, z \in \mathbb{D}$, without using any additional assumptions.

Example 1 Let $f_{1}, f_{2} \in H^{\infty}$ be linearly independent solutions of (1) for $A \in H_{2}^{\infty}$. This example concerns different situations that may happen.

(i) There are a lot of examples in which $\inf _{z \in \mathbb{D}}\left(\left|f_{1}(z)\right|+\left|f_{2}(z)\right|\right)>0$. See the discussion after the proof of [11, Theorem 2], for example.

(ii) The proof of Theorem 3 below produces an example, where the condition (12) holds; take $\left\{z_{n}\right\}$ as in Theorem 3 and note that $\left|f_{1}\right|+\left|f_{2}\right| \geq\left|f_{1}\right|$, where $f_{1}$ has the desired property. At the same time, $\left|f_{1}\left(z_{n}\right)\right|+\left|f_{2}\left(z_{n}\right)\right| \asymp 1-\left|z_{n}\right|^{2}$ as $n \rightarrow \infty$. Not only $\inf _{z \in \mathbb{D}}\left(\left|f_{1}(z)\right|+\left|f_{2}(z)\right|\right)>0$ fails to be true but also it breaks down in the worst possible way.

(iii) Let $f_{1}(z)=\left(1-z^{2}\right)^{1 / 2}$ and $f_{2}(z)=\left(1-z^{2}\right)^{1 / 2} \log ((1+z) /(1-z)), z \in \mathbb{D}$. These functions are linearly independent solutions of (1) for the coefficient $A(z)=1 /\left(1-z^{2}\right)^{2}$, $z \in \mathbb{D}$, which evidently satisfies $A \in H_{2}^{\infty}$. Since both solutions have radial limit zero along the positive real axis, the condition (12) cannot hold for any pairwise disjoint pseudo-hyperbolic discs.

\section{Proofs of Theorems 3 and 4}

The first part of the proof of Theorem 3 follows directly from that of [11, Corollary 3]. The new contribution lies in the fact that the differential equation in question admits only bounded solutions.

Proof (of Theorem 3) Let $B=B_{\Lambda}$ be the Blaschke product corresponding to the uniformly separated sequence $\Lambda=\left\{z_{n}\right\}$. By (20) and Cauchy's integral formula, $\sup _{z_{n} \in \Lambda}\left|B^{\prime \prime}\left(z_{n}\right)\right| /\left|B^{\prime}\left(z_{n}\right)\right|^{2}<\infty$. Let $f_{1}=B e^{B k}$, where $k \in H^{\infty}$ is a solution of the interpolation problem

$$
k\left(z_{n}\right)=-\frac{B^{\prime \prime}\left(z_{n}\right)}{2 B^{\prime}\left(z_{n}\right)^{2}}, \quad z_{n} \in \Lambda .
$$

As in the proof of Theorem 15, the coefficient $A=-f_{1}^{\prime \prime} / f_{1} \in \operatorname{Hol}(\mathbb{D})$ induces a Carleson measure $|A(z)|^{2}\left(1-|z|^{2}\right)^{3} d m(z)$. Now, $f_{1}$ is a solution of (1) which has precisely the prescribed zeros $\Lambda$.

Since $\Lambda$ is uniformly separated, there exists a constant $0<\delta<1$ such that $\Omega=$ $\bigcup_{z_{n} \in \Lambda} \Delta_{p}\left(z_{n}, \delta\right)$ is a union of pairwise disjoint pseudo-hyperbolic discs. Fix any $\alpha \in \mathbb{D} \backslash \Omega$, and define the meromorphic function $f_{2}$ by

$$
f_{2}(z)=f_{1}(z) \int_{\alpha}^{z} \frac{1}{f_{1}(\zeta)^{2}} d \zeta, \quad z \in \mathbb{D} .
$$


Choose the path of integration by the following rules. If $z \in \mathbb{D} \backslash \Omega$, then the whole path lies in $\mathbb{D} \backslash \Omega$. If $z \in \Delta_{p}\left(z_{n}, \delta\right)$ for some $z_{n} \in \Lambda$, then the path stays in $(\mathbb{D} \backslash \Omega) \cup \Delta_{p}\left(z_{n}, \delta\right)$. Then, each point $z \in \mathbb{D}$ can be reached by a path which satisfies these properties and is also of uniformly bounded Euclidean length. The following argument is standard. In a sufficiently small pseudo-hyperbolic neighborhood of $\alpha, f_{2}$ represents an analytic function such that $f_{1} f_{2}^{\prime}-f_{1}^{\prime} f_{2}$ is identically one. As a solution of (1) function $f_{2}$ admits an analytic continuation to $\mathbb{D}$, and this continuation agrees with the representation (19).

There exists a constant $\mu=\mu(\Lambda)$ such that $|B(\zeta)| \geq \mu>0$ for $\zeta \in \mathbb{D} \backslash \Omega$; see [4, Theorem 1] for example. We deduce

$$
\left|f_{2}(z)\right| \leq|B(z)| e^{|B(z)||k(z)|} \int_{\alpha}^{z} \frac{|d \zeta|}{|B(\zeta)|^{2} e^{-2|B(\zeta)||k(\zeta)|}} \leq \frac{e^{3\|k\|_{H} \infty}}{\mu^{2}} \int_{\alpha}^{z}|d \zeta|,
$$

for $z \in \mathbb{D} \backslash \Omega$. Lemma 1 implies that $f_{2} \in H^{\infty}$. Since $W\left(f_{1}, f_{2}\right)=1$, we obtain

$$
\begin{aligned}
\left(f_{1} / f_{2}\right)^{\#}\left(z_{n}\right)\left(1-\left|z_{n}\right|^{2}\right)^{2} & =\frac{1}{\left|f_{2}\left(z_{n}\right)\right|^{2}}\left(1-\left|z_{n}\right|^{2}\right)^{2}=\left|f_{1}^{\prime}\left(z_{n}\right)\right|^{2}\left(1-\left|z_{n}\right|^{2}\right)^{2} \\
& =\left|B^{\prime}\left(z_{n}\right)\right|^{2}\left(1-\left|z_{n}\right|^{2}\right)^{2}, \quad z_{n} \in \Lambda .
\end{aligned}
$$

This completes the proof as $\Lambda$ is uniformly separated.

The proof of Theorem 4 depends on a supporting result, which is considered next. Suppose that $f \in \operatorname{Hol}(\mathbb{D}), f: \mathbb{D} \rightarrow \mathbb{D}, f(0)=0$ and $\left|f^{\prime}(0)\right| \geq \delta$ for some $0<\delta \leq 1$. By Cauchy's integral formula and Schwarz's lemma,

$$
\left|f^{\prime}(0)\right|-\left|f^{\prime}(z)\right| \leq \frac{12|z|}{(1-|z|)^{2}}, \quad z \in \mathbb{D} .
$$

If $0<\eta<1$ satisfies $12 \eta /(1-\eta)^{2}<\delta / 2$, then $\left|f^{\prime}(z)\right| \geq \delta / 2$ for all $|z|<\eta$. The following lemma is a conformally invariant version of this property.

Lemma 2 Suppose that $f \in \operatorname{Hol}(\mathbb{D})$ and $f: \mathbb{D} \rightarrow \mathbb{D}$. Assume that there exists a sequence $\Lambda \subset \mathbb{D}$ such that inf $z_{n} \in \Lambda\left|f^{\prime}\left(z_{n}\right)\right|\left(1-\left|z_{n}\right|^{2}\right) \geq \delta>0$. If $0<\eta<1$ satisfies $12 \eta /(1-\eta)^{2}<$ $\delta / 2$, then there exist a constant $v=v(\delta)$ such that

$$
\left|f^{\prime}(z)\right|\left(1-|z|^{2}\right) \geq v>0, \quad z \in \bigcup_{z_{n} \in \Lambda} \Delta_{p}\left(z_{n}, \eta\right) .
$$

Proof Let $z_{n} \in \Lambda$ be fixed, and define $g_{z_{n}}=\varphi_{f\left(z_{n}\right)} \circ f \circ \varphi_{z_{n}}$. Now $g_{z_{n}}: \mathbb{D} \rightarrow \mathbb{D}$ is analytic, $g_{z_{n}}(0)=0$, and

$$
\left|g_{z_{n}}^{\prime}(0)\right|=\left|\varphi_{f\left(z_{n}\right)}^{\prime}\left(f\left(z_{n}\right)\right)\right|\left|f^{\prime}\left(z_{n}\right)\right|\left(1-\left|z_{n}\right|^{2}\right) \geq\left|f^{\prime}\left(z_{n}\right)\right|\left(1-\left|z_{n}\right|^{2}\right) \geq \delta .
$$

The property above implies

$$
\left|g_{z_{n}}^{\prime}(z)\right|=\left|\varphi_{f\left(z_{n}\right)}^{\prime}\left(f\left(\varphi_{z_{n}}(z)\right)\right)\right| \cdot\left|f^{\prime}\left(\varphi_{z_{n}}(z)\right)\right| \cdot\left|\varphi_{z_{n}}^{\prime}(z)\right| \geq \delta / 2, \quad|z|<\eta .
$$

If we denote $w=\varphi_{z_{n}}(z)$, then $|z|<\eta$ if and only if $w \in \Delta_{p}\left(z_{n}, \eta\right)$. Consequently,

$$
\left|f^{\prime}(w)\right|\left(1-|w|^{2}\right) \geq \frac{\delta}{2} \cdot \frac{1-\left|\varphi_{z_{n}}(w)\right|^{2}}{1-\left|\varphi_{f\left(z_{n}\right)}(f(w))\right|^{2}}\left(1-|f(w)|^{2}\right), \quad w \in \Delta\left(z_{n}, \eta\right) .
$$

Since $\varrho_{p}\left(w, z_{n}\right)<\eta$, we have $\varrho_{p}\left(f(w), f\left(z_{n}\right)\right)<\eta$ by Schwarz's lemma. Therefore there exists a constant $\delta^{\star}=\delta^{\star}(\delta, \eta)>0$ such that

$$
\left|f^{\prime}(w)\right|\left(1-|w|^{2}\right) \geq \delta^{\star}\left(1-\left|f\left(z_{n}\right)\right|^{2}\right) \geq \delta^{\star}\left|f^{\prime}\left(z_{n}\right)\right|\left(1-\left|z_{n}\right|^{2}\right) \geq \delta^{\star} \delta, \quad w \in \Delta\left(z_{n}, \eta\right),
$$


by the Schwarz-Pick lemma. The claim follows for $v=\delta^{\star} \delta$.

Proof (of Theorem 4) The proof is divided into two steps. The first step takes advantage of two results concerning interpolation in $H^{\infty}$.

Construction of auxiliary functions. Let $B=B_{\Lambda}$ be the Blaschke product corresponding to the uniformly separated sequence $\Lambda=\left\{z_{n}\right\}$, and let $\left\{w_{n}\right\}$ be the bounded target sequence for the desired interpolation. Consequently,

$$
\inf _{z_{n} \in \Lambda}\left|B^{\prime}\left(z_{n}\right)\right|\left(1-\left|z_{n}\right|^{2}\right)=\inf _{z_{n} \in \Lambda} \prod_{z_{k} \in \Lambda \backslash\left\{z_{n}\right\}}\left|\frac{z_{k}-z_{n}}{1-\bar{z}_{k} z_{n}}\right|=\delta>0 .
$$

Let $0<\eta<1$ satisfy $12 \eta /(1-\eta)^{2}<\delta / 2$. Then, in particular, $\eta<\delta / 3$. Earl's interpolation theorem [6, Theorem 2], applied with $\eta$ instead of $\delta$, shows that

$$
\left\{h \in H^{\infty}: h\left(z_{n}\right)=w_{n} \text { for all } z_{n} \in \Lambda\right\}
$$

can be solved by a constant multiple of a Blaschke product. More precisely, there exist $C=C\left(\Lambda,\left\{w_{n}\right\}, \eta\right) \in \mathbb{C}$ and a Blaschke product $I=I\left(\Lambda,\left\{w_{n}\right\}, \eta\right)$ such that

(i) $h=C I$ solves the interpolation problem (21);

(ii) the zeros $\Lambda^{\star}=\left\{\zeta_{n}\right\}$ of $I=I_{\left\{\zeta_{n}\right\}}$ satisfy $\zeta_{n} \in \Delta_{p}\left(z_{n}, \eta\right)$ for all $n$.

The standard estimates show that

$$
\inf _{\zeta_{n} \in \Lambda^{\star}}\left|I^{\prime}\left(\zeta_{n}\right)\right|\left(1-\left|\zeta_{n}\right|^{2}\right)=\inf _{\zeta_{n} \in \Lambda^{\star}} \prod_{\zeta_{k} \in \Lambda^{\star} \backslash\left\{\zeta_{n}\right\}}\left|\frac{\zeta_{k}-\zeta_{n}}{1-\bar{\zeta}_{k} \zeta_{n}}\right| \geq \frac{\delta}{3}>0,
$$

and therefore $\left\{\zeta_{n}\right\}$ is also uniformly separated.

By applying Lemma 2 to the Blaschke product $B$, there exists another constant $v$ such that $\left|B^{\prime}\left(\zeta_{n}\right)\right|\left(1-\left|\zeta_{n}\right|^{2}\right) \geq v>0$ for all $\zeta_{n} \in \Lambda^{\star}$. According to Øyma's interpolation theorem [37, Theorem 1], there exists $g \in H^{\infty}$ such that

$$
g\left(\zeta_{n}\right)=-\frac{I^{\prime \prime}\left(\zeta_{n}\right)}{2 I^{\prime}\left(\zeta_{n}\right) B^{\prime}\left(\zeta_{n}\right)}, \quad g^{\prime}\left(\zeta_{n}\right)=0, \quad \zeta_{n} \in \Lambda^{\star}
$$

Note that the target sequence for $g$ is bounded by the obtained estimates.

Construction of the differential equation. Let $f=C I e^{B g} \in \operatorname{Hol}(\mathbb{D})$, where $C \in \mathbb{C}$ and $I, B, g$ are functions as in the above construction. Clearly, $f\left(z_{n}\right)=w_{n}$ for all $z_{n} \in \Lambda$. The zeros of $f$ are precisely the points in $\Lambda^{\star}$, and they are pairwise pseudo-hyperbolically close to the corresponding points in $\Lambda$ by (ii). Since

$$
f^{\prime \prime}\left(\zeta_{n}\right)=C e^{B\left(\zeta_{n}\right) g\left(\zeta_{n}\right)}\left(I^{\prime \prime}\left(\zeta_{n}\right)+2 I^{\prime}\left(\zeta_{n}\right) B^{\prime}\left(\zeta_{n}\right) g\left(\zeta_{n}\right)\right)=0, \quad \zeta_{n} \in \Lambda^{\star}
$$

the function

$$
A=-\frac{f^{\prime \prime}}{f}=-\frac{I^{\prime \prime}+2 I^{\prime}(B g)^{\prime}}{I}-\left((B g)^{\prime}\right)^{2}-(B g)^{\prime \prime},
$$

is analytic in $\mathbb{D}$. More precisely, the points in $\Lambda^{\star}$ are removable singularities for the coefficient $A$ as $g$ solves the interpolation problem (22). As in the proof of Theorem 15, we conclude that $|A(z)|^{2}\left(1-|z|^{2}\right)^{3} d m(z)$ is a Carleson measure. The fact that all solutions of (1) are bounded follows as in the proof of Theorem 3. This completes the proof of Theorem 4. 


\subsection{Separation of zeros and critical points}

Let $A \in H_{2}^{\infty}$, and let $f$ be a non-trivial solution of (1). By [43, Theorem 3], the zeros of $f$ are separated in the hyperbolic metric by a constant depending only on $\|A\|_{H_{2}^{\infty}}$, and by [10, Corollary 2], the hyperbolic distance between any zero and any critical point of $f$ is uniformly bounded away from zero in a similar fashion. Moreover, [10, Example 1] shows that critical points of $f$ need not to obey any kind of separation. The situation becomes more difficult if we consider similar questions between zeros and critical points of linearly independent solutions. See [10, Section 4] for related discussion.

The following result concerns differential equations with bounded solutions. The proof is based on an auxiliary estimate [5, Lemma 7, p. 209]: If $f \in H_{\alpha}^{\infty}$ for $0 \leq \alpha<\infty$, then there exists a constant $C=C(\alpha)$ with $0<C<\infty$ such that

$$
|| f\left(z_{1}\right)\left|\left(1-\left|z_{1}\right|^{2}\right)^{\alpha}-\right| f\left(z_{2}\right)\left|\left(1-\left|z_{2}\right|^{2}\right)^{\alpha}\right| \leq C \varrho_{p}\left(z_{1}, z_{2}\right)\|f\|_{H_{\alpha}^{\infty}}
$$

for all points $z_{1}, z_{2} \in \mathbb{D}$ with $\varrho_{p}\left(z_{1}, z_{2}\right) \leq 1 / 2$. The sharpness discussion of Proposition 3 below is omitted.

Proposition 3 Suppose that $A \in \operatorname{Hol}(\mathbb{D})$ and all solutions of (1) are bounded.

(i) It is possible that for each $0<\delta<1$ there exists a solution of (1), depending on $\delta$, which has two distinct zeros $z_{1}, z_{2} \in \mathbb{D}$ such that $\varrho_{p}\left(z_{1}, z_{2}\right)<\delta$.

(ii) Critical points of non-trivial solutions are not separated in any way.

Let $f_{1}, f_{2} \in H^{\infty}$ be linearly independent solutions of (1).

(iii) If $z_{1} \in \mathbb{D}$ is a zero and $z_{2} \in \mathbb{D}$ is a critical point of $f_{1}$, then there exists a constant $0<C<\infty$ such that

$$
\varrho_{p}\left(z_{1}, z_{2}\right) \geq C \frac{\left|W\left(f_{1}, f_{2}\right)\right|}{\left\|f_{1}\right\|_{H^{\infty}}\left\|f_{2}\right\|_{H^{\infty}}} \max \left\{1-\left|z_{1}\right|, 1-\left|z_{2}\right|\right\} .
$$

(iv) If $z_{1} \in \mathbb{D}$ is a zero of $f_{1}$, and $z_{2} \in \mathbb{D}$ is a zero of $f_{2}$, then (24) holds.

(v) If $z_{1} \in \mathbb{D}$ is a critical point of $f_{1}$, and $z_{2} \in \mathbb{D}$ is that of $f_{2}$, then (24) holds.

Proof (i) Let the coefficient $A \in \operatorname{Hol}(\mathbb{D}) \backslash H_{2}^{\infty}$ be as in Theorem 1(i). If the pseudo-hyperbolic distance between any distinct zeros of any non-trivial solution of (1) is uniformly bounded away from zero, then $A \in H_{2}^{\infty}$ by [43, Theorem 4]. This is a contradiction, and therefore (i) holds in this particular case.

(ii) The assertion follows from [10, Example 1], since in this example all solutions of (1) are bounded; use (9) to obtain a bounded linearly independent solution.

(iii) Let $f_{1} \in H^{\infty}$ be the non-trivial solution of (1) with $f_{1}\left(z_{1}\right)=0=f_{1}^{\prime}\left(z_{2}\right)$. If $\varrho_{p}\left(z_{1}, z_{2}\right)>1 / 2$, then there is nothing to prove. Otherwise, let $f_{2} \in H^{\infty}$ be a solution of (1), which is linearly independent to $f_{1}$. Since $f_{1}\left(z_{2}\right) f_{2}^{\prime}\left(z_{2}\right)=W\left(f_{1}, f_{2}\right)$, there exists a constant $0<C_{1}<\infty$ such that

$$
\varrho_{p}\left(z_{1}, z_{2}\right) \geq \frac{\left|f_{1}\left(z_{2}\right)\right|}{C_{1}\left\|f_{1}\right\|_{H^{\infty}}}=\frac{\left|W\left(f_{1}, f_{2}\right)\right|}{C_{1}\left\|f_{1}\right\|_{H^{\infty}}\left|f_{2}^{\prime}\left(z_{2}\right)\right|} \geq \frac{\left|W\left(f_{1}, f_{2}\right)\right|\left(1-\left|z_{2}\right|^{2}\right)}{C_{1}\left\|f_{1}\right\|_{H^{\infty}}\left\|f_{2}\right\|_{H^{\infty}}}
$$

by (23); note that $\left\|f_{2}^{\prime}\right\|_{H_{1}^{\infty}} \leq\left\|f_{2}\right\|_{H^{\infty}}$ by standard estimates. Analogously, since $-f_{1}^{\prime}\left(z_{1}\right) f_{2}\left(z_{1}\right)=W\left(f_{1}, f_{2}\right)$, there exists another constant $0<C_{2}<\infty$ such that

$$
\varrho_{p}\left(z_{1}, z_{2}\right) \geq \frac{\left|f_{1}^{\prime}\left(z_{1}\right)\right|\left(1-\left|z_{1}\right|^{2}\right)}{C_{2}\left\|f_{1}^{\prime}\right\|_{H_{1}^{\infty}}}=\frac{\left|W\left(f_{1}, f_{2}\right)\right|\left(1-\left|z_{1}\right|^{2}\right)}{C_{2}\left\|f_{1}^{\prime}\right\|_{H_{1}^{\infty}}\left|f_{2}\left(z_{1}\right)\right|} \geq \frac{\left|W\left(f_{1}, f_{2}\right)\right|\left(1-\left|z_{1}\right|^{2}\right)}{C_{2}\left\|f_{1}\right\|_{H^{\infty}}\left\|f_{2}\right\|_{H^{\infty}}} .
$$


Statements (iv) and (v) are proved similarly. In the case of (iv) apply (23) to $f_{1}, f_{2} \in H^{\infty}$, and in the case of (v) apply (23) to $f_{1}^{\prime}, f_{2}^{\prime} \in H_{1}^{\infty}$.

\section{Proof of Theorem 5}

After the proof of Theorem 5, we consider its relation to conformal metrics of constant curvature. We also discuss an application concerning Carleson measures induced by bounded solutions of (1) for $A \in \operatorname{Hol}(\mathbb{D})$.

Proof (of Theorem 5) It is clear that $u$ is sufficiently smooth to be in the class $C^{2}$.

(i) Since $\left(f_{1} / f_{2}\right)^{\prime}=-W\left(f_{1}, f_{2}\right) / f_{2}^{2}$, we deduce

$$
\left(\frac{f_{1}}{f_{2}}\right)^{\#}=\frac{\left|W\left(f_{1}, f_{2}\right)\right|}{\left|f_{1}\right|^{2}+\left|f_{2}\right|^{2}}, \quad \partial u=\frac{f_{1}^{\prime} \overline{f_{1}}+f_{2}^{\prime} \overline{f_{2}}}{\left|f_{1}\right|^{2}+\left|f_{2}\right|^{2}} .
$$

We compute

$$
\Delta u=4 \bar{\partial}(\partial u)=4 \frac{\left|f_{1} f_{2}^{\prime}-f_{1}^{\prime} f_{2}\right|^{2}}{\left(\left|f_{1}\right|^{2}+\left|f_{2}\right|^{2}\right)^{2}}=4 e^{-2 u} .
$$

(ii) As above, we obtain

$$
\begin{aligned}
\frac{1}{4} \Delta u & =\partial(\bar{\partial} u)=\frac{\left|f_{1}^{\prime}\right|^{2}+\left|f_{2}^{\prime}\right|^{2}}{\left|f_{1}\right|^{2}+\left|f_{2}\right|^{2}}-\frac{f_{1} \overline{f_{1}^{\prime}}+f_{2} \overline{f_{2}^{\prime}}}{\left|f_{1}\right|^{2}+\left|f_{2}\right|^{2}} \cdot \frac{f_{1}^{\prime} \overline{f_{1}}+f_{2}^{\prime} \overline{f_{2}}}{\left|f_{1}\right|^{2}+\left|f_{2}\right|^{2}} \\
& =\frac{\left|f_{1}^{\prime}\right|^{2}+\left|f_{2}^{\prime}\right|^{2}}{\left|f_{1}\right|^{2}+\left|f_{2}\right|^{2}}-(\bar{\partial} u) \cdot(\partial u) .
\end{aligned}
$$

Since $u$ is real-valued, $\Delta u=\left(\Delta e^{u}\right) /\left(e^{u}\right)-4|\partial u|^{2}=\left(\Delta e^{u}\right) /\left(e^{u}\right)-|\nabla u|^{2}$.

(iii) We deduce

$$
\partial^{2} u=\frac{f_{1}^{\prime \prime} \bar{f}_{1}+f_{2}^{\prime \prime} \bar{f}_{2}}{\left|f_{1}\right|^{2}+\left|f_{2}\right|^{2}}-(\partial u)^{2}=-\frac{A\left|f_{1}\right|^{2}+A\left|f_{2}\right|^{2}}{\left|f_{1}\right|^{2}+\left|f_{2}\right|^{2}}-(\partial u)^{2}=-A-(\partial u)^{2},
$$

which completes the proof.

Remark 1 Let $f_{1}$ and $f_{2}$ be linearly independent solutions of (1) for $A \in \operatorname{Hol}(\mathbb{D})$. As in the proof of Theorem 5(i), we deduce that $v=-u=\log \left(f_{1} / f_{2}\right)^{\#}$ is a solution of the Liouville equation $\Delta v=-4 e^{2 v}$. Recall that $\lambda(z)|d z|$ is said to be a conformal metric on $\mathbb{D}$ if the conformal density $\lambda: \mathbb{D} \rightarrow \mathbb{R}$ is strictly positive and continuous. If $\lambda \in C^{2}$, then $\lambda(z)|d z|$ is called a regular conformal metric on $\mathbb{D}$. The (Gauss) curvature $\kappa: \mathbb{D} \rightarrow \mathbb{R}$ of the regular conformal metric $\lambda(z)|d z|$ is given by $\kappa=-\Delta(\log \lambda) / \lambda^{2}$. In conclusion, $\left(f_{1} / f_{2}\right)^{\#}(z)|d z|$ defines a regular conformal metric of constant curvature 4 on $\mathbb{D}$.

As an application of Theorem 5, we return to consider differential equations with bounded solutions. Theorem 3 shows that, even if $f_{1}, f_{2} \in H^{\infty}$ are linearly independent solutions of (1) for $A \in \operatorname{Hol}(\mathbb{D})$, it may happen that $f_{1} / f_{2}$ is non-normal and $\left(\left(f_{1} / f_{2}\right)^{\#}\right)^{2} \log (1 /|z|) d m(z)$ is not a Carleson measure. The following result and Theorem 5(ii) imply that this Carleson measure condition becomes true if the exponent 2 is replaced by any smaller value.

Theorem 16 Let $f_{1}, f_{2} \in H^{\infty}$ be linearly independent solutions of $(1)$ for $A \in \operatorname{Hol}(\mathbb{D})$. Then, $\left(\left|f_{1}^{\prime}\right|^{2}+\left|f_{2}^{\prime}\right|^{2}\right)\left(\left|f_{1}\right|^{2}+\left|f_{2}\right|^{2}\right)^{\varepsilon-1} \log (1 /|z|) d m(z)$ is a Carleson measure for any $0<\varepsilon<\infty$. 
Let $\Omega \subset \mathbb{R}^{2}$ be a domain with smooth boundary, and let $u_{1}, u_{2}$ be $C^{2}$-functions on $\bar{\Omega}$. The classical Green theorem asserts

$$
\int_{\partial \Omega}\left(u_{1} \frac{\partial u_{2}}{\partial n}-u_{2} \frac{\partial u_{1}}{\partial n}\right) d s=\int_{\Omega}\left(u_{1} \Delta u_{2}-u_{2} \Delta u_{1}\right) d x d y,
$$

where $\partial / \partial n$ denotes differentiation in the direction of outward pointing normal and $d s$ is the arc length on $\partial \Omega$. The following argument is based on a modification of Uchiyama's lemma. We refer to [36, p. 290] and [46, Lemma 2.1] for the original statement. Suppose that $f \in \operatorname{Hol}(\mathbb{D})$ and $\varphi \in C^{2}$ is a subharmonic function in $\mathbb{D}$. By the theorems of Green and Fubini, we deduce

$$
\begin{aligned}
& \frac{1}{2 \pi} \int_{0}^{2 \pi} e^{\varphi\left(r e^{i \theta}\right)}\left|f\left(r e^{i \theta}\right)\right|^{2} d \theta-e^{\varphi(0)}|f(0)|^{2} \\
& \quad=\frac{1}{2 \pi} \int_{D(0, r)} \Delta\left(e^{\varphi}|f|^{2}\right)(z) \log \frac{r}{|z|} d m(z)
\end{aligned}
$$

for any $0<r<1$. Since

$$
\left|\zeta_{1}+\zeta_{2}\right|^{2} \geq\left(\left|\zeta_{1}\right|-\left|\zeta_{2}\right|\right)^{2} \geq \frac{1}{2}\left|\zeta_{1}\right|^{2}-\left|\zeta_{2}\right|^{2}, \quad \zeta_{1}, \zeta_{2} \in \mathbb{C}
$$

we obtain

$$
\Delta\left(e^{\varphi}|f|^{2}\right)=e^{\varphi}(\Delta \varphi)|f|^{2}+4 e^{\varphi}\left|(\partial \varphi) f+f^{\prime}\right|^{2} \geq e^{\varphi}\left(\Delta \varphi+2|\partial \varphi|^{2}\right)|f|^{2}-4 e^{\varphi}\left|f^{\prime}\right|^{2},
$$

and finally

$$
\begin{aligned}
& \frac{1}{2 \pi} \int_{D(0, r)}|f(z)|^{2} e^{\varphi(z)}\left(\Delta \varphi(z)+2|\partial \varphi(z)|^{2}\right) \log \frac{r}{|z|} d m(z) \\
& \quad \leq \frac{1}{2 \pi} \int_{0}^{2 \pi} e^{\varphi\left(r e^{i \theta}\right)}\left|f\left(r e^{i \theta}\right)\right|^{2} d \theta+\frac{2}{\pi} \int_{D(0, r)} e^{\varphi(z)}\left|f^{\prime}(z)\right|^{2} \log \frac{r}{|z|} d m(z)
\end{aligned}
$$

for any $0<r<1$.

Proof (of Theorem 16) Let $f_{1}, f_{2} \in H^{\infty}$ be linearly independent solutions of (1) for $A \in$ $\operatorname{Hol}(\mathbb{D})$. Without loss of generality, we may assume that $W\left(f_{1}, f_{2}\right)=1$. We conclude that $\varphi=\varepsilon u=\varepsilon \log \left(\left|f_{1}\right|^{2}+\left|f_{2}\right|^{2}\right)$ is bounded from above and subharmonic in $\mathbb{D}$, as $\Delta \varphi=$ $4 \varepsilon\left(\left(f_{1} / f_{2}\right)^{\#}\right)^{2} \geq 0$ by Theorem 5(i). By the Littlewood-Paley formula [8, Lemma 3.1], we obtain

$$
\|f\|_{H^{2}}^{2}=|f(0)|^{2}+\frac{2}{\pi} \int_{\mathbb{D}}\left|f^{\prime}(z)\right|^{2} \log \frac{1}{|z|} d m(z), \quad f \in \operatorname{Hol}(\mathbb{D}),
$$

and therefore a standard convergence argument applied to (26) reveals that

$$
\begin{aligned}
& \frac{1}{2 \pi} \int_{\mathbb{D}}|f(z)|^{2} e^{\varphi(z)}\left(\Delta \varphi(z)+2|\partial \varphi(z)|^{2}\right) \log \frac{1}{|z|} d m(z) \\
& \quad \leq 2\left(\left\|f_{1}\right\|_{H^{\infty}}^{2}+\left\|f_{2}\right\|_{H^{\infty}}^{2}\right)^{\varepsilon}\|f\|_{H^{2}}^{2}
\end{aligned}
$$

for any $f \in H^{2}$. This proves that $e^{\varphi(z)}\left(\Delta \varphi(z)+2|\partial \varphi(z)|^{2}\right) \log (1 /|z|) d m(z)$ is a Carleson measure, and therefore by Theorem 5(ii), we deduce

$$
\begin{aligned}
e^{\varphi}\left(\Delta \varphi+2|\partial \varphi|^{2}\right) & =\left(\left|f_{1}\right|^{2}+\left|f_{2}\right|^{2}\right)^{\varepsilon}\left(\varepsilon \Delta u+\frac{\varepsilon^{2}}{2}|\nabla u|^{2}\right) \\
& \geq \min \left\{\varepsilon, \varepsilon^{2} / 2\right\}\left(\left|f_{1}\right|^{2}+\left|f_{2}\right|^{2}\right)^{\varepsilon} \frac{\Delta e^{u}}{e^{u}} .
\end{aligned}
$$


This completes the proof of Theorem 16 .

\section{Proofs of Theorem 6 and Proposition 1}

Recall that the meromorphic function $g$ in the unit disc belongs to the Nevanlinna class $\mathcal{N}$ if and only if the Ahlfors-Shimizu characteristic

$$
T_{0}(r, g)=\frac{1}{\pi} \int_{0}^{r}\left(\int_{D(0, t)} g^{\#}(z)^{2} d m(z)\right) \frac{d t}{t}=\frac{1}{\pi} \int_{D(0, r)} g^{\#}(z)^{2} \log \frac{r}{|z|} d m(z)
$$

is uniformly bounded for $0<r<1$. The equivalence of the representations above follows from Fubini's theorem.

Let $u \not \equiv-\infty$ be subharmonic in $\mathbb{D}$. Function $u$ admits a harmonic majorant in $\mathbb{D}$ if and only if $\lim _{r \rightarrow 1^{-}} \int_{0}^{2 \pi} u\left(r e^{i \theta}\right) d \theta<\infty$, and in this case, the least harmonic majorant for $u$ is

$$
\hat{u}(z)=\lim _{r \rightarrow 1^{-}} \frac{1}{2 \pi} \int_{0}^{2 \pi} u\left(r e^{i \theta}\right) \frac{r^{2}-|z|^{2}}{\left|r e^{i \theta}-z\right|^{2}} d \theta<\infty, \quad z \in \mathbb{D} .
$$

See [41, Theorem 3.3] for more details. In the proof of Theorem 6 we take advantage of the following well-known fact: If $u \in C^{2}$ is subharmonic and $\varphi$ is analytic, then $u \circ \varphi$ is subharmonic with $\Delta(u \circ \varphi)=((\Delta u) \circ \varphi)\left|\varphi^{\prime}\right|^{2}$.

Proof (of Theorem 6) (i) By Green's theorem (25) with $u_{1}=1, u_{2}=u$, we obtain

$$
\frac{d}{d t} \int_{0}^{2 \pi} u\left(t e^{i \theta}\right) d \theta=\frac{4}{t} \int_{D(0, t)}\left(\left(f_{1} / f_{2}\right)^{\#}(z)\right)^{2} d m(z), \quad 0<t<1,
$$

as $\Delta u=4\left(\left(f_{1} / f_{2}\right)^{\#}\right)^{2}$ by Theorem 5(i). By integrating from 0 to $r$, we conclude $1 /(2 \pi) \int_{0}^{2 \pi} u\left(r e^{i \theta}\right) d \theta=u(0)+2 T_{0}\left(r, f_{1} / f_{2}\right)$ for any $0<r<1$. Consequently, $u$ admits a harmonic majorant if and only if $f_{1} / f_{2} \in \mathcal{N}$.

(ii) Let $a \in \mathbb{D}$. By Green's theorem and Theorem 5(i),

$$
\begin{aligned}
\frac{1}{2 \pi} & \int_{0}^{2 \pi} u\left(a+(1-|a|) r e^{i \theta}\right) d \theta-u(a) \\
& =\frac{2}{\pi} \int_{0}^{r}\left(\int_{D(a, t(1-|a|))}\left(f_{1} / f_{2}\right)^{\#}(z)^{2} d m(z)\right) \frac{d t}{t}, \quad 0<r<1 .
\end{aligned}
$$

By letting $r \rightarrow 1^{-}$, we deduce

$$
\sup _{a \in \mathbb{D}} \widehat{u_{a}}(0)=\sup _{a \in \mathbb{D}} \frac{2}{\pi} \int_{0}^{1}\left(\int_{D(a, t(1-|a|))}\left(f_{1} / f_{2}\right)^{\#}(z)^{2} d m(z)\right) \frac{d t}{t} .
$$

This completes the proof of (ii), as $f_{1} / f_{2}$ is a normal function in the Nevanlinna class if and only if the right-hand side of (27) is finite [38, Theorem 1].

(iii) The assertion is in some sense a meromorphic counterpart of [47, Theorem 5.1]. Fix $a \in \mathbb{D}$, and take $r$ to be sufficiently large to satisfy $|a|<r<1$. Define $\psi(z)=r \varphi_{a / r}(z / r)$, $z \in \mathbb{D}$. By Green's theorem,

$$
\begin{aligned}
\frac{1}{2 \pi} \int_{0}^{2 \pi} u\left(r e^{i \theta}\right) \frac{r^{2}-|a|^{2}}{\left|r e^{i \theta}-a\right|^{2}} d \theta-u(a) & =\frac{1}{2 \pi} \int_{0}^{2 \pi}(u \circ \psi)\left(r e^{i t}\right) d t-(u \circ \psi)(0) \\
& =\frac{1}{2 \pi} \int_{D(0, r)} \Delta u(z) \log \frac{1}{\left|\varphi_{a / r}(z / r)\right|} d m(z) .
\end{aligned}
$$


By using standard estimates and letting $r \rightarrow 1^{-}$, we conclude that $\hat{u}(a)-u(a) \asymp$ $\int_{\mathbb{D}} \Delta u(z)\left(1-\left|\varphi_{a}(z)\right|^{2}\right) d m(z)$, where the comparison constants are independent of $a \in \mathbb{D}$. Theorem 5(i) implies

$$
\sup _{a \in \mathbb{D}}(\hat{u}(a)-u(a)) \asymp \sup _{a \in \mathbb{D}} \int_{\mathbb{D}}\left(\left(f_{1} / f_{2}\right)^{\#}(z)\right)^{2}\left(1-|z|^{2}\right) \frac{1-|a|^{2}}{|1-\bar{a} z|^{2}} d m(z) .
$$

The part (iii) follows as $f_{1} / f_{2} \in \mathrm{UBC}$ if and only if $\left(\left(f_{1} / f_{2}\right)^{\#}(z)\right)^{2}\left(1-|z|^{2}\right) d m(z)$ is a Carleson measure [38, Theorem 3].

The proofs of (iv)-(vi) are straight-forward and hence omitted. Note that the function $e^{u}=\left(\left|f_{1}\right|^{2}+\left|f_{2}\right|^{2}\right) /\left|W\left(f_{1}, f_{2}\right)\right|$ is subharmonic in $\mathbb{D}$.

It is well-known that non-trivial solutions of a Blaschke-oscillatory equation (1), $A \in$ $\operatorname{Hol}(\mathbb{D})$, may lie outside the Nevanlinna class $\mathcal{N}$ [22, Section 4.3]. In the following remark, we deduce an estimate according to which the Nevanlinna characteristic of solutions of Blaschke-oscillatory equations cannot grow arbitrarily fast.

Remark 2 Let $f_{1}$ be a non-trivial solution of a Blaschke-oscillatory equation (1) for $A \in$ $\operatorname{Hol}(\mathbb{D})$. Let $f_{2}$ be another solution of $(1)$, which is linearly independent to $f_{1}$. Note that $f_{2} / f_{1} \in \mathcal{N}$ by [22, Lemma 3], and $\left(f_{2} / f_{1}\right)^{\prime}=W\left(f_{1}, f_{2}\right) / f_{1}^{2}$ by straight-forward computation. Kennedy's estimate [25, Theorem 1] implies

$$
S=\int_{0}^{1}(1-r) e^{2 T\left(r,\left(f_{2} / f_{1}\right)^{\prime}\right)} d r<\infty .
$$

Nevanlinna's first theorem shows that (28) remains to be true, if $T\left(r,\left(f_{2} / f_{1}\right)^{\prime}\right)$ is replaced by $2 T\left(r, f_{1}\right)$. This places a severe restriction for the growth of $T\left(r, f_{1}\right)$ as $r \rightarrow 1^{-}$. It implies $T\left(r, f_{1}\right) \leq(1 / 2) \log (\sqrt{2 S} /(1-r))$ for all $0<r<1$. Therefore all solutions of (1) are non-admissible [22, p. 53].

Proof (of Proposition 1) Recall that $\left(f_{1} / f_{2}\right)^{\#}=\left|W\left(f_{1}, f_{2}\right)\right| /\left(\left|f_{1}\right|^{2}+\left|f_{2}\right|^{2}\right)$. Now

$$
\begin{aligned}
\int_{\mathbb{D}}( & \left.\left(f_{1} / f_{2}\right)^{\#}\right)^{2}\left(1-|z|^{2}\right) d m(z) \\
\leq & \frac{\left|W\left(f_{1}, f_{2}\right)\right|^{2}}{\delta^{2}} \int_{\left\{z \in \mathbb{D}:\left|f_{1}(z)\right|^{2}+\left|f_{2}(z)\right|^{2} \geq \delta\right\}}\left(1-|z|^{2}\right) d m(z) \\
& \quad+\left(\sup _{z \in \mathbb{D}}\left(f_{1} / f_{2}\right)^{\#}(z)^{2}\left(1-|z|^{2}\right)^{2}\right) \int_{\left\{z \in \mathbb{D}:\left|f_{1}(z)\right|^{2}+\left|f_{2}(z)\right|^{2}<\delta\right\}} \frac{d m(z)}{1-|z|^{2}} .
\end{aligned}
$$

Therefore $f_{1} / f_{2}$ belongs to the Nevanlinna class by the assumption.

We briefly consider two applications of Proposition 1 . Suppose that $f_{1}, f_{2}$ are linearly independent solutions of (1) for $A \in \operatorname{Hol}(\mathbb{D})$ and assume that (12) holds for some Blaschke sequence $\left\{z_{n}\right\} \subset \mathbb{D}$ and $0<\delta<1$. Denote this infimum by $0<s<\infty$. We deduce

$$
\begin{aligned}
\int_{\left\{z \in \mathbb{D}:\left|f_{1}(z)\right|^{2}+\left|f_{2}(z)\right|^{2}<s^{2} / 2\right\}} \frac{d m(z)}{1-|z|^{2}} & \leq \sum_{n} \int_{\Delta_{p}\left(z_{n}, \delta\right)} \frac{d m(z)}{1-|z|^{2}} \\
& \asymp \sum_{n}\left(1-\left|z_{n}\right|\right)<\infty,
\end{aligned}
$$

where the pseudo-hyperbolic discs $\Delta_{p}\left(z_{n}, \delta\right)$ are not necessarily pairwise disjoint. In such a case the normality of $f_{1} / f_{2}$ implies that $f_{1} / f_{2} \in \mathcal{N}$ by Proposition 1 . 
The same conclusion is obtained if $f_{1} / f_{2}$ is normal and $\left|f_{1}\right|+\left|f_{2}\right|$ is uniformly bounded from below for all points in $\mathbb{D}$ which lie outside a horodisc (that is, a disc internally tangent to $\partial \mathbb{D})$. The details are omitted.

\section{Proof of Theorem 7}

We begin with a lemma, which is needed in the proof of Theorem 7. This auxiliary result is based on the well-known Harnack inequalities: if $h \in \mathrm{Har}^{+}(\mathbb{D})$, then

$$
\frac{1-\varrho_{p}(z, w)}{1+\varrho_{p}(z, w)} \leq \frac{h(z)}{h(w)} \leq \frac{1+\varrho_{p}(z, w)}{1-\varrho_{p}(z, w)}, \quad z, w \in \mathbb{D} .
$$

Let $f \in \operatorname{Hol}(\mathbb{D})$ and recall that $f \in \mathcal{N}$ if and only if there exists $h \in \operatorname{Har}^{+}(\mathbb{D})$ such that $\log ^{+}|f| \leq h$, which is equivalent to the fact $|f| \leq e^{h}$. There is no reason to expect that any order derivative of $f$ would belong to $\mathcal{N}$. However, for every $k \in \mathbb{N}$, there exists a constant $C=C(k)$ with $0<C<\infty$ such that

$$
\left|f^{(k)}(z)\right|\left(1-|z|^{2}\right)^{k} \leq e^{C h(z)}, \quad z \in \mathbb{D},
$$

by Cauchy's integral formula and Harnack's inequality. See [19, Lemma 2.1].

Lemma 3 Suppose that $f \in \operatorname{Hol}(\mathbb{D})$ and it satisfies $|f(z)|\left(1-|z|^{2}\right)^{k} \leq e^{h(z)}, z \in \mathbb{D}$, for $k \in \mathbb{N} \cup\{0\}$ and $h \in \operatorname{Har}^{+}(\mathbb{D})$. If $f$ vanishes on a sequence $\Lambda \in \operatorname{Int} \mathcal{N}$, then there exists $H \in \operatorname{Har}^{+}(\mathbb{D})$ such that $|f(z)|\left(1-|z|^{2}\right)^{k} \leq \varrho_{p}(\Lambda, z) e^{H(z)}, z \in \mathbb{D}$.

Proof Consider a dyadic partition of $\mathbb{D}$ into Whitney squares of the type

$$
Q=Q_{I}=\{z \in \mathbb{D}: 1-|I| /(2 \pi) \leq|z|<1, \arg z \in I\}
$$

where $\ell(Q)=|I|$ is the arc-length of the interval $I \subset \partial \mathbb{D}$. The top part of $Q$ is $T(Q)=$ $\{z \in Q: 1-\ell(Q) /(2 \pi) \leq|z| \leq 1-\ell(Q) /(4 \pi)\}$.

Let $Q$ be any Whitney square in the dyadic partition. Let $\Omega_{1} \subset \mathbb{D}$ such that

$$
T(Q) \subset \Omega_{1}, \quad \varrho_{p}\left(\partial \Omega_{1}, \partial T(Q)\right)=\operatorname{diam}_{p}(T(Q)),
$$

and let $\Omega_{2}$ be another set such that $\Omega_{1} \subset \Omega_{2} \subset \mathbb{D}$ and $\varrho_{p}\left(\partial \Omega_{2}, \partial \Omega_{1}\right)=4$ diam $_{p} \Omega_{1}$. Here $\operatorname{diam}_{p}$ denotes the pseudo-hyperbolic diameter. Define $g \in \mathcal{H}(\mathbb{D})$ by

$$
g(z)=f(z)\left(\prod_{z_{k} \in \Lambda \cap \Omega_{1}} \frac{z_{k}-z}{1-\bar{z}_{k} z}\right)^{-1}, \quad z \in \mathbb{D} .
$$

We may assume that $\Lambda \cap \Omega_{1}$ is not empty, for otherwise the assertion follows for all $z \in T(Q)$ by trivial reasons. Fix any $z_{n} \in \Lambda \cap \Omega_{1}$. We deduce

$$
|g(\zeta)| \leq \frac{\left(1-|\zeta|^{2}\right)^{-k} e^{h(\zeta)}}{\varrho_{p}\left(z_{n}, \zeta\right)}\left(\prod_{z_{k} \in \Lambda \cap \Omega_{1}: z_{k} \neq z_{n}}\left|\frac{z_{k}-\zeta}{1-\bar{z}_{k} \zeta}\right|\right)^{-1}, \quad \zeta \in \partial \Omega_{2} .
$$

Since $\Lambda \in \operatorname{Int} \mathcal{N}$, [18, Theorem 1.2] implies that there exists $h_{1} \in \operatorname{Har}^{+}(\mathbb{D})$ with

$$
|g(\zeta)| \lesssim\left(1-|\zeta|^{2}\right)^{-k} e^{h(\zeta)+h_{1}\left(z_{n}\right)} \lesssim\left(1-\left|z_{n}\right|^{2}\right)^{-k} e^{\left(C h+h_{1}\right)\left(z_{n}\right)}, \quad \zeta \in \partial \Omega_{2},
$$


where $0<C<\infty$ is a universal constant by Harnack's inequalities. The maximum modulus principle extends this estimate for all $z \in \Omega_{2}$, and therefore

$$
|f(z)| \leq|g(z)| \prod_{z_{k} \in \Lambda \cap \Omega_{1}}\left|\frac{z_{k}-z}{1-\bar{z}_{k} z}\right| \lesssim\left(1-\left|z_{n}\right|^{2}\right)^{-k} e^{\left(C h+h_{1}\right)\left(z_{n}\right)} \varrho_{p}(\Lambda, z),
$$

for $z \in T(Q)$. By Harnack's inequalities, there exists $H \in \operatorname{Har}^{+}(\mathbb{D})$ such that the assertion holds for all $z \in T(Q)$. Since the argument is independent of the Whitney square $Q$, the proof is complete.

Proof (of Theorem 7) Let $B=B_{\Lambda}$ be the Blaschke product with zeros $\Lambda \in \operatorname{Int} \mathcal{N}$ and let $f=B e^{B g}$, where $g \in \operatorname{Hol}(\mathbb{D})$ is a solution of the interpolation problem

$$
g\left(z_{n}\right)=w_{n}, \quad w_{n}=-\frac{B^{\prime \prime}\left(z_{n}\right)}{2\left(B^{\prime}\left(z_{n}\right)\right)^{2}}, \quad z_{n} \in \Lambda .
$$

As $\Lambda \in \operatorname{Int} \mathcal{N}$, [18, Theorem 1.2] implies that there exists $h_{1} \in \mathrm{Har}^{+}(\mathbb{D})$ with

$$
\left|B^{\prime}\left(z_{n}\right)\right|\left(1-\left|z_{n}\right|^{2}\right)=\prod_{z_{k} \in \Lambda: z_{k} \neq z_{n}}\left|\frac{z_{k}-z_{n}}{1-\bar{z}_{k} z_{n}}\right| \geq e^{-h_{1}\left(z_{n}\right)}, \quad z_{n} \in \Lambda .
$$

Since there exists a constant $0<C<\infty$ such that

$$
\log ^{+}\left|w_{n}\right|=\log ^{+}\left|\frac{B^{\prime \prime}\left(z_{n}\right)}{2\left(B^{\prime}\left(z_{n}\right)\right)^{2}}\right| \leq C+2 h_{1}\left(z_{n}\right), \quad z_{n} \in \mathbb{D},
$$

[18, Theorem 1.2] ensures that $\left\{w_{n}\right\} \in \mathcal{N} \mid \Lambda$. Therefore we may assume $g \in \mathcal{N}$.

By straight-forward computation, $f$ is a solution of (1) for $A \in \operatorname{Hol}(\mathbb{D})$, where

$$
A=-\frac{f^{\prime \prime}}{f}=-\frac{B^{\prime \prime}+2 B^{\prime}\left(B^{\prime} g+B g^{\prime}\right)}{B}-\left((B g)^{\prime}\right)^{2}-(B g)^{\prime \prime} .
$$

The interpolation property (30) guarantees that every point $z_{n} \in \Lambda$ is a removable singularity for $A$. It remains to show that there exists $h \in \operatorname{Har}^{+}(\mathbb{D})$ such that $|A(z)|\left(1-|z|^{2}\right)^{2} \leq e^{h(z)}$, $z \in \mathbb{D}$. Since $B g \in \mathcal{N},(29)$ implies that the two right-most terms in (32) are of the desired type. Since $B^{\prime \prime}+2 B^{\prime}\left(B^{\prime} g+B g^{\prime}\right)$ vanishes on the sequence $\Lambda$, Lemma 3 shows that there exists $h_{2} \in \mathrm{Har}^{+}(\mathbb{D})$ such that

$$
\left|B^{\prime \prime}(z)+2 B^{\prime}(z)\left(B^{\prime}(z) g(z)+B(z) g^{\prime}(z)\right)\right|\left(1-|z|^{2}\right)^{2} \leq \varrho_{p}(\Lambda, z) e^{h_{2}(z)}, \quad z \in \mathbb{D} .
$$

And finally, by [19, Theorem 1.2], there exists $h_{3} \in \operatorname{Har}^{+}(\mathbb{D})$ such that $|B(z)| \geq$ $\varrho_{p}(\Lambda, z) e^{-h_{3}(z)}, z \in \mathbb{D}$. We deduce Theorem 7 by combining the estimates.

\section{Proof of Theorem 8}

The following result is an analogue of Carleson's [2, Theorem 2], which characterizes those cases in which the classical 0, 1-interpolation is possible. The proof of Proposition 4 is based on the Nevanlinna corona theorem by Mortini [33, Satz 4]: Given $f_{1}, f_{2} \in \mathcal{N}$, the Bézout equation $f_{1} g_{2}+f_{2} g_{2}=1$ can be solved with functions $g_{1}, g_{2} \in \mathcal{N}$ if and only if there exists $h \in \operatorname{Har}^{+}(\mathbb{D})$ such that $\left|f_{1}(z)\right|+\left|f_{2}(z)\right| \geq e^{-h(z)}, z \in \mathbb{D}$. 
Proposition 4 Let $\left\{z_{n}\right\},\left\{\zeta_{n}\right\}$ be Blaschke sequences. Then, there exists $f \in \mathcal{N}$ such that $f\left(z_{n}\right)=0$ and $f\left(\zeta_{n}\right)=1$ for all $n$ if and only if there exists $h \in \operatorname{Har}^{+}(\mathbb{D})$ such that (6) holds.

Proof Assume that there exists $f \in \mathcal{N}$ such that $f\left(z_{n}\right)=0$ and $f\left(\zeta_{n}\right)=1$ for all $n$. By the classical factorization theorem, there exist functions $g_{1}, g_{2} \in \mathcal{N}$ such that $f=B_{\left\{z_{n}\right\}} g_{1}=$ $1+B_{\left\{\zeta_{n}\right\}} g_{2}$. Here $B_{\left\{z_{n}\right\}}$ and $B_{\left\{\zeta_{n}\right\}}$ are Blaschke products with zeros $\left\{z_{n}\right\}$ and $\left\{\zeta_{n}\right\}$, respectively. As $g_{1}, g_{2} \in \mathcal{N}$, there exist $h_{1}, h_{2} \in \operatorname{Har}^{+}(\mathbb{D})$ such that $\left|g_{1}\right| \leq e^{h_{1}}$ and $\left|g_{2}\right| \leq e^{h_{2}}$. We deduce

$$
1=\left|B_{\left\{z_{n}\right\}} g_{1}-B_{\left\{\zeta_{n}\right\}} g_{2}\right| \leq e^{h_{1}+h_{2}}\left(\left|B_{\left\{z_{n}\right\}}\right|+\left|B_{\left\{\zeta_{n}\right\}}\right|\right),
$$

which proves the first part of the assertion.

Assume that there exists $h \in \mathrm{Har}^{+}(\mathbb{D})$ such that (6) holds. By the Nevanlinna corona theorem, there exist $g_{1}, g_{2} \in \mathcal{N}$ such that $B_{\left\{z_{n}\right\}} g_{1}+B_{\left\{\zeta_{n}\right\}} g_{2}=1$. Then, the function $f=$ $B_{\left\{z_{n}\right\}} g_{1} \in \mathcal{N}$ satisfies the desired 0, 1-interpolation.

Proof (of Theorem 8) By Proposition 4, there exists $g \in \mathcal{N}$ such that $g\left(z_{n}\right)=0$ and $g\left(\zeta_{n}\right)=1$ for all $n$. Now $f(z)=\exp (\log \alpha+g(z) \log (\beta / \alpha)), z \in \mathbb{D}$, satisfies the desired interpolation property, and is a zero-free solution of (1) for $A \in \operatorname{Hol}(\mathbb{D})$,

$$
A(z)=-\frac{f^{\prime \prime}(z)}{f(z)}=-\left(g^{\prime}(z) \log \frac{\beta}{\alpha}\right)^{2}-g^{\prime \prime}(z) \log \frac{\beta}{\alpha}, \quad z \in \mathbb{D} .
$$

By (29), there exists $H \in \operatorname{Har}^{+}(\mathbb{D})$ such that $|A(z)|\left(1-|z|^{2}\right)^{2} \leq e^{H(z)}, z \in \mathbb{D}$.

\section{Proofs of Theorems 9 and 10, and Proposition 2}

The following proof proceeds along the same lines as that in [44, p. 129].

Proof (of Theorem 9) If $f_{1}, f_{2}$ are linearly independent solutions of (1) for $A \in \operatorname{Hol}(\mathbb{D})$, then

$$
W\left(f_{1}, f_{2}\right) A=f_{1}^{\prime} f_{2}^{\prime \prime}-f_{1}^{\prime \prime} f_{2}^{\prime}, \quad\left(f_{1} / f_{2}\right)^{\#}=\left|W\left(f_{1}, f_{2}\right)\right| /\left(\left|f_{1}\right|^{2}+\left|f_{2}\right|^{2}\right) .
$$

Since $W\left(f_{1}, f_{2}\right)$ is a non-zero complex constant, the estimate (29) and the fact $f_{1}, f_{2} \in$ $\mathcal{N}$ imply that there exists $h_{1} \in \operatorname{Har}^{+}(\mathbb{D})$ such that $|A(z)|\left(1-|z|^{2}\right)^{3} \leq e^{h_{1}(z)}, z \in \mathbb{D}$. Moreover, the Cauchy-Schwarz inequality (18) and the estimate (29) show that there exists $h_{2} \in \operatorname{Har}^{+}(\mathbb{D})$ such that $\left(f_{1} / f_{2}\right)^{\#}(z)\left(1-|z|^{2}\right)^{2} \leq e^{h_{2}(z)}, z \in \mathbb{D}$. The claim follows by choosing $H=h_{1}+h_{2} \in \operatorname{Har}^{+}(\mathbb{D})$.

The proof of Theorem 10 is analogous to that proof of Theorem 2, which is presented in the end of Sect. 4.

Proof (of Theorem 10) By (7) and [19, Theorem 1], the ideal $I_{\mathcal{N}}\left(f_{1}, f_{2}\right)$ contains a Blaschke product $B$ whose zero-sequence belongs to $\operatorname{Int} \mathcal{N}$. This is equivalent to the fact that there exist functions $g_{1}, g_{2} \in \mathcal{N}$ such that $f_{1} g_{1}+f_{2} g_{2}=B$. Differentiate $f_{1} g_{1}+f_{2} g_{2}=B$ twice, and apply (1) to $f_{1}^{\prime \prime}$ and $f_{2}^{\prime \prime}$ to obtain (17). Note that $A \in \operatorname{Hol}(\mathbb{D})$ by assumption. As in the proof of Theorem 7, we conclude that there exists $H \in \operatorname{Har}^{+}(\mathbb{D})$ such that $\sup _{z \in \mathbb{D}}|A(z)|\left(1-|z|^{2}\right)^{2} \leq$ $e^{H(z)}, z \in \mathbb{D}$. 
Proof (of Proposition 2) Proposition 2 follows directly from [3, Theorem 15] if $\psi$ : $\mathbb{D} \rightarrow(0,1 / 2)$ given by $\psi(z)=e^{-H(z) / 2} e^{-1}, z \in \mathbb{D}$ and $H \in \operatorname{Har}^{+}(\mathbb{D})$, satisfies $\sup _{a, z \in \mathbb{D}} \psi(a) / \psi\left(\varphi_{a}(\psi(a) z)\right)<\infty$. Now

$$
\begin{aligned}
\sup _{a, z \in \mathbb{D}} & \exp \left(\frac{H(a)}{2}\left(\frac{H\left(\varphi_{a}\left(e^{-H(a) / 2} e^{-1} z\right)\right)}{H\left(\varphi_{a}(0)\right)}-1\right)\right) \\
\leq & \sup _{a, z \in \mathbb{D}} \exp \left(\frac{H(a)}{2}\left(\frac{1+\varrho_{p}\left(0, e^{-H(a) / 2} e^{-1} z\right)}{1-\varrho_{p}\left(0, e^{-H(a) / 2} e^{-1} z\right)}-1\right)\right)
\end{aligned}
$$

by Harnack's inequalities. This is bounded by

$$
\sup _{0 \leq x<\infty} \exp \left(\frac{x}{2}\left(\frac{1+e^{-x / 2} e^{-1}}{1-e^{-x / 2} e^{-1}}-1\right)\right)<\frac{3}{2},
$$

which implies the assertion.

\subsection{Separation of zeros and critical points}

We proceed to state an analogue of Proposition 3. If $f \in \operatorname{Hol}(\mathbb{D})$ and

$$
\|f\|=\sup _{z \in \mathbb{D}}|f(z)|\left(1-|z|^{2}\right)^{\alpha} e^{-h(z)}<\infty
$$

for $0 \leq \alpha<\infty$ and $h \in \operatorname{Har}^{+}(\mathbb{D})$, then there exists $C=C(\alpha)>0$ such that

$$
|| f\left(z_{1}\right)\left|\left(1-\left|z_{1}\right|^{2}\right)^{\alpha} e^{-h\left(z_{1}\right)}-\right| f\left(z_{2}\right)\left|\left(1-\left|z_{2}\right|^{2}\right)^{\alpha} e^{-h\left(z_{2}\right)}\right| \leq C \varrho_{p}\left(z_{1}, z_{2}\right)\|f\|,
$$

for all points $z_{1}, z_{2} \in \mathbb{D}$ with $\varrho_{p}\left(z_{1}, z_{2}\right) \leq 1 / 2$. This estimate follows immediately from (23): If $f \in \operatorname{Hol}(\mathbb{D})$ satisfies (33) for $0 \leq \alpha<\infty$ and $h \in \mathrm{Har}^{+}(\mathbb{D})$, then (23) can be applied to $f e^{-h-i h^{\star}} \in H_{\alpha}^{\infty}$, where $h^{\star}$ is a harmonic conjugate of $h$.

Proposition 5 Let $f_{1}, f_{2} \in \mathcal{N}$ be linearly independent solutions of (1) for $A \in \operatorname{Hol}(\mathbb{D})$.

(i) If $z_{1} \in \mathbb{D}$ is a zero and $z_{2} \in \mathbb{D}$ is a critical point of $f_{1}$, then there exists $h \in \operatorname{Har}^{+}(\mathbb{D})$ such that

$$
\varrho_{p}\left(z_{1}, z_{2}\right) \gtrsim \max \left\{\left(1-\left|z_{1}\right|\right) e^{-h\left(z_{1}\right)},\left(1-\left|z_{2}\right|\right) e^{-h\left(z_{2}\right)}\right\} .
$$

(ii) If $z_{1} \in \mathbb{D}$ is a zero of $f_{1}$, and $z_{2} \in \mathbb{D}$ is a zero of $f_{2}$, then there exists $h \in \operatorname{Har}^{+}(\mathbb{D})$ such that (34) holds.

(iii) If $z_{1} \in \mathbb{D}$ is a critical point of $f_{1}$, and $z_{2} \in \mathbb{D}$ is a critical point of $f_{2}$, then there exists $h \in \operatorname{Har}^{+}(\mathbb{D})$ such that (34) holds.

The proof of Proposition 5 is omitted.

\section{Proofs of Theorem 11 and Corollary 2}

The proof of Theorem 11 is based on a smoothness property, which is considered first. Let $\omega$ be a radial weight on $\mathbb{D}$. Then,

$$
\varrho_{\omega}\left(z_{1}, z_{2}\right)=\int_{\left\langle z_{1}, z_{2}\right\rangle} \frac{|d z|}{\omega(z)}, \quad z_{1}, z_{2} \in \mathbb{D},
$$


defines a distance function. Here, we integrate along the hyperbolic segment $\left\langle z_{1}, z_{2}\right\rangle$ between the points $z_{1}, z_{2} \in \mathbb{D}$, where the hyperbolic segment is a closed subset of the corresponding hyperbolic geodesic. For $\omega(z)=1-|z|^{2}, z \in \mathbb{D}$, the function $\varrho_{\omega}$ reduces to the standard hyperbolic distance $\varrho_{h}$ :

$$
\varrho_{h}\left(z_{1}, z_{2}\right)=\frac{1}{2} \log \frac{1+\varrho_{p}\left(z_{1}, z_{2}\right)}{1-\varrho_{p}\left(z_{1}, z_{2}\right)}, \quad \varrho_{p}\left(z_{1}, z_{2}\right)=\left|\frac{z_{2}-z_{1}}{1-\bar{z}_{2} z_{1}}\right|, \quad z_{1}, z_{2} \in \mathbb{D} .
$$

Lemma 4 Let $f_{1}, f_{2}$ be linearly independent solutions of (1) for $A \in \operatorname{Hol}(\mathbb{D})$, and define $u=-\log \left(f_{1} / f_{2}\right)^{\#}$. Let $\omega$ be a radial weight. If

$$
\sup _{z \in \mathbb{D}}|\nabla u(z)| \omega(z) \leq \Lambda<\infty
$$

then

$$
e^{-\Lambda \varrho_{\omega}\left(z_{1}, z_{2}\right)} \leq \frac{\left|f_{1}\left(z_{1}\right)\right|^{2}+\left|f_{2}\left(z_{1}\right)\right|^{2}}{\left|f_{1}\left(z_{2}\right)\right|^{2}+\left|f_{2}\left(z_{2}\right)\right|^{2}} \leq e^{\Lambda \varrho_{\omega}\left(z_{1}, z_{2}\right)}, \quad z_{1}, z_{2} \in \mathbb{D} .
$$

Conversely, if (36) holds for some constant $0<\Lambda<\infty$, then (35) is satisfied.

Proof Assume that (35) holds. Let $z_{1}, z_{2} \in \mathbb{D}$ be distinct points, and let $\gamma=\gamma(t), 0 \leq t \leq 1$, be a parametrization of $\left\langle z_{1}, z_{2}\right\rangle$. Schwarz's inequality and (35) imply

$$
\begin{aligned}
\left|\log \frac{\left|f_{1}\left(z_{1}\right)\right|^{2}+\left|f_{2}\left(z_{1}\right)\right|^{2}}{\left|f_{1}\left(z_{2}\right)\right|^{2}+\left|f_{2}\left(z_{2}\right)\right|^{2}}\right| & =\left|u\left(z_{1}\right)-u\left(z_{2}\right)\right| \leq\left|\int_{0}^{1} \nabla u(\gamma(t)) \cdot \gamma^{\prime}(t) d t\right| \\
& \leq \int_{0}^{1}|\nabla u(\gamma(t))|\left|\gamma^{\prime}(t)\right| d t \leq \Lambda \varrho_{\omega}\left(z_{1}, z_{2}\right) .
\end{aligned}
$$

From this estimate we deduce (36).

Assume that (36) holds for some constant $0<\Lambda<\infty$. Fix $z_{2} \in \mathbb{D}$. Since

$$
\lim _{z_{1} \rightarrow z_{2}} \frac{\left|z_{1}-z_{2}\right|}{\varrho_{h}\left(z_{1}, z_{2}\right)}=\lim _{z_{1} \rightarrow z_{2}} \frac{\varrho_{p}\left(z_{1}, z_{2}\right)}{\frac{1}{2} \log \frac{1+\varrho_{p}\left(z_{1}, z_{2}\right)}{1-\varrho_{p}\left(z_{1}, z_{2}\right)}} \cdot\left|1-\bar{z}_{1} z_{2}\right|=1-\left|z_{2}\right|^{2},
$$

and

$$
\frac{\left|z_{1}-z_{2}\right|}{\varrho_{h}\left(z_{1}, z_{2}\right)} \cdot \frac{1}{\max _{z \in\left\langle z_{1}, z_{2}\right\rangle \frac{1-|z|^{2}}{\omega(z)}}} \leq \frac{\left|z_{1}-z_{2}\right|}{\varrho_{\omega}\left(z_{1}, z_{2}\right)} \leq \frac{\left|z_{1}-z_{2}\right|}{\varrho_{h}\left(z_{1}, z_{2}\right)} \cdot \frac{1}{\min _{z \in\left\langle z_{1}, z_{2}\right\rangle} \frac{1-|z|^{2}}{\omega(z)}}
$$

for any $z_{1} \in \mathbb{D}$, we conclude that $\lim _{z_{1} \rightarrow z_{2}}\left|z_{1}-z_{2}\right| / \varrho_{\omega}\left(z_{1}, z_{2}\right)=\omega\left(z_{2}\right)$ by the continuity of $\omega$. Therefore,

$$
\left|\nabla u\left(z_{2}\right)\right| \omega\left(z_{2}\right)=\lim _{z_{1} \rightarrow z_{2}}\left|\frac{u\left(z_{1}\right)-u\left(z_{2}\right)}{z_{1}-z_{2}}\right| \frac{\left|z_{1}-z_{2}\right|}{\varrho_{\omega}\left(z_{1}, z_{2}\right)} \leq \lim _{z_{1} \rightarrow z_{2}} \frac{\Lambda \varrho_{\omega}\left(z_{1}, z_{2}\right)}{\varrho_{\omega}\left(z_{1}, z_{2}\right)}=\Lambda .
$$

This completes the proof of Lemma 4.

The following lemma is important for our cause due to the representation (13).

Lemma 5 Let $f_{1}, f_{2}$ be linearly independent solutions of (1) for $A \in \operatorname{Hol}(\mathbb{D})$, and define $u=-\log \left(f_{1} / f_{2}\right)^{\#}$. Suppose that $\omega$ is a regular weight which satisfies $\sup _{z \in \mathbb{D}} \omega(z) /(1-$ $|z|)<\infty$. If $|\nabla u| \in L_{\omega}^{\infty}$, then

$$
\frac{\left|f_{1}^{(j)}\right|+\left|f_{2}^{(j)}\right|}{\left|f_{1}\right|+\left|f_{2}\right|} \in L_{\omega^{j}}^{\infty}, \quad j \in \mathbb{N} .
$$


Proof By the assumption, there exists a positive constant $c$ such that the discs $\mathfrak{D}(z)=$ $D(z, c \omega(z))$ satisfy $\mathfrak{D}(z) \subset D(z,(1-|z|) / 2), z \in \mathbb{D}$. Let $\zeta \in \partial \mathfrak{D}(z)$. Since $\langle z, \zeta\rangle \subset$ $D(z,(1-|z|) / 2)$, a straight-forward argument based on (8) reveals

$$
\varrho_{\omega}(z, \zeta) \lesssim \frac{1-|z|^{2}}{\omega(z)} \varrho_{h}(z, \zeta) \lesssim \frac{1-|z|^{2}}{\omega(z)} \varrho_{p}(z, \zeta) \lesssim \frac{|z-\zeta|}{\omega(z)}=c .
$$

Therefore $\sup _{z \in \mathbb{D}} \max _{\zeta \in \partial \mathfrak{D}(z)} \varrho_{\omega}(z, \zeta)<\infty$.

By Cauchy's integral formula,

$$
\begin{aligned}
\left|f_{1}^{(j)}(z)\right|+\left|f_{2}^{(j)}(z)\right| & \leq 2 \max \left\{\left|f_{1}^{(j)}(z)\right|,\left|f_{2}^{(j)}(z)\right|\right\} \\
& \leq\left(\max _{\zeta \in \partial \mathfrak{D}(z)}\left(\left|f_{1}(\zeta)\right|+\left|f_{2}(\zeta)\right|\right)\right) \frac{2 j !}{c^{j} \omega(z)^{j}}, \quad z \in \mathbb{D} .
\end{aligned}
$$

Now (37) and Lemma 4 imply

$$
\begin{aligned}
\frac{\left|f_{1}^{(j)}(z)\right|+\left|f_{2}^{(j)}(z)\right|}{\left|f_{1}(z)\right|+\left|f_{2}(z)\right|} & \leq \frac{2 j ! \sqrt{2}}{c^{j} \omega(z)^{j}}\left(\max _{\zeta \in \partial \mathfrak{D}(z)} \frac{\left|f_{1}(\zeta)\right|^{2}+\left|f_{2}(\zeta)\right|^{2}}{\left|f_{1}(z)\right|^{2}+\left|f_{2}(z)\right|^{2}}\right)^{1 / 2} \\
& \leq \frac{2 j ! \sqrt{2}}{c^{j} \omega(z)^{j}} \exp \left(\frac{\||\nabla u|\|_{L_{\omega}^{\infty}}}{2} \max _{\zeta \in \partial \mathfrak{D}(z)} \varrho_{\omega}(z, \zeta)\right) \lesssim \frac{1}{\omega(z)^{j}}
\end{aligned}
$$

for $z \in \mathbb{D}$. The assertion of Lemma 5 follows.

Finally, proceed to prove Theorem 11. We take advantage of Yamashita's [48, Corollary to Theorem 2, p. 161], which uses the following notation. For a meromorphic function $f$ and $z \in \mathbb{D}$, let $\rho(z, f)$ be the maximum of $0<r \leq 1$ such that $f$ is univalent in $\Delta_{p}(z, r)$, and let $\rho_{a}(z, f)$ be the maximum of $0<r \leq 1$ such that $f(w) \neq-1 / \overline{f(z)}$ for all $w \in \Delta_{p}(z, r)$. Note that $-1 / \overline{f(z)}$ is the antipodal point of $f(z)$ in the Riemann sphere.

Proof (of Theorem 11) First, assume that $|\nabla u| \in L_{\omega}^{\infty}$. By the representation (13) and Lemma 5, we conclude that $A \in H_{\omega^{2}}^{\infty}$. By Theorem 5(i) and (ii),

$$
4\left(\left(f_{1} / f_{2}\right)^{\#}\right)^{2}=\Delta u \leq \frac{\Delta e^{u}}{e^{u}}=\frac{\left|f_{1}^{\prime}\right|^{2}+\left|f_{2}^{\prime}\right|^{2}}{\left|f_{1}\right|^{2}+\left|f_{2}\right|^{2}} \leq 2\left(\frac{\left|f_{1}^{\prime}\right|+\left|f_{2}^{\prime}\right|}{\left|f_{1}\right|+\left|f_{2}\right|}\right)^{2},
$$

and therefore $\left(f_{1} / f_{2}\right)^{\#} \in L_{\omega}^{\infty}$ by Lemma 5 .

Second, let $A \in H_{\omega^{2}}^{\infty}$ and $\left(f_{1} / f_{2}\right)^{\#} \in L_{\omega}^{\infty}$. Since $f_{1} / f_{2}$ is meromorphic in $\mathbb{D}$ and has zero-free spherical derivative, Yamashita's [48, Corollary to Theorem 2, p. 161] implies

$$
\left(1-|z|^{2}\right)\left|\frac{-\bar{z}}{1-|z|^{2}}-\partial u(z)\right| \leq \frac{2}{\min \left\{\rho\left(z, f_{1} / f_{2}\right), \rho_{a}\left(z, f_{1} / f_{2}\right)\right\}}, \quad z \in \mathbb{D} .
$$

We deduce

$$
|\nabla u(z)| \leq \frac{2}{1-|z|^{2}}\left(1+\frac{2}{\min \left\{\rho\left(z, f_{1} / f_{2}\right), \rho_{a}\left(z, f_{1} / f_{2}\right)\right\}}\right), \quad z \in \mathbb{D} .
$$

Denote $h=f_{1} / f_{2}$. It suffices to show that both $\rho(z, h)$ and $\rho_{a}(z, h)$ are bounded from below by a constant multiple of $\omega(z) /\left(1-|z|^{2}\right)$ as $|z| \rightarrow 1^{-}$.

Let $\psi: \mathbb{D} \rightarrow(0, \infty)$ be the weight $\psi(z)=c \omega(z) /\left(1-|z|^{2}\right)$, where $0<c<1$ is a sufficiently small constant whose value is determined later. By the assumption, we may 
assume that $\psi: \mathbb{D} \rightarrow(0,1 / 2)$ and therefore $\varphi_{a}(\psi(a) z) \in \Delta_{p}(a, 1 / 2)$ for all $a, z \in \mathbb{D}$. By (8) and standard estimates,

$$
\sup _{a, z \in \mathbb{D}} \frac{\psi(a)}{\psi\left(\varphi_{a}(\psi(a) z)\right)}=\sup _{a, z \in \mathbb{D}} \frac{\omega(a)}{\omega\left(\varphi_{a}(\psi(a) z)\right)} \cdot \frac{1-\left|\varphi_{a}(\psi(a) z)\right|^{2}}{1-|a|^{2}}<\infty .
$$

Function $h$ is locally univalent and meromorphic, and its Schwarzian derivative satisfies $S_{h}=2 A$. Let $g_{a}(z)=\left(h \circ \varphi_{a}\right)(\psi(a) z)$ for $a, z \in \mathbb{D}$. By the chain rule,

$$
\begin{aligned}
\left|S_{g_{a}}(z)\right| & =\left|S_{h}\left(\varphi_{a}(\psi(a) z)\right)\right|\left|\varphi_{a}^{\prime}(\psi(a) z)\right|^{2} \psi(a)^{2} \\
& \leq \frac{2\|A\|_{H_{\omega^{2}}^{\infty}}}{\omega\left(\varphi_{a}(\psi(a) z)\right)^{2}} \frac{\left(1-\left|\varphi_{a}(\psi(a) z)\right|^{2}\right)^{2}}{\left(1-|\psi(a) z|^{2}\right)^{2}} \frac{c^{2} \omega(a)^{2}}{\left(1-|a|^{2}\right)^{2}}, \quad a, z \in \mathbb{D} .
\end{aligned}
$$

We deduce that $\left\|S_{g_{a}}\right\|_{H^{\infty}} \leq \pi^{2} / 2$ for any $a \in \mathbb{D}$, provided that $0<c<1$ is sufficiently small. Therefore $g_{a}$ is univalent in the unit disc [34, Theorem II] for any $a \in \mathbb{D}$. This is equivalent to the fact that $h$ is univalent in $\Delta_{p}(a, \psi(a))$ for any $a \in \mathbb{D}$, and therefore $\rho(a, h) \geq \psi(a)$ for $a \in \mathbb{D}$.

It remains to estimate $\rho_{a}(z, h)$. Let $\sigma$ denote the spherical distance on the Riemann sphere. By the assumption $h^{\#} \in L_{\omega}^{\infty}$, we obtain

$$
\begin{aligned}
\sigma(h(z), h(\zeta)) & \leq \int_{h(\langle z, \zeta\rangle)} \frac{|d \xi|}{1+|\xi|^{2}}=\int_{\langle z, \zeta\rangle} h^{\#}(\xi)|d \xi| \\
& \leq\left(\sup _{\xi \in\langle z, \zeta\rangle} \frac{1-|\xi|^{2}}{\omega(\xi)}\right) \varrho_{h}(z, \zeta)
\end{aligned}
$$

for any $z, \zeta \in \mathbb{D}$. If $\zeta \in \Delta_{p}(z, \psi(z))$, which is a subset of $\Delta_{p}(z, 1 / 2)$, then

$$
\sigma(h(z), h(\zeta)) \leq\left(\sup _{\xi \in\langle z, \zeta\rangle} \frac{1-|\xi|^{2}}{\omega(\xi)}\right) 2 \varrho_{p}(z, \zeta) \lesssim \frac{1-|z|^{2}}{\omega(z)} \cdot \frac{c \omega(z)}{1-|z|^{2}}
$$

with an absolute comparison constant. Then, $h(z)$ and $h(\zeta)$ cannot be antipodal points if $0<c<1$ is sufficiently small. Therefore $\rho_{a}(z, h) \geq \psi(z)$ for $z \in \mathbb{D}$, which completes the proof of Theorem 11 .

Corollary 1 allows us to reach the desired conclusion $A \in H_{2}^{\infty}$ under the assumption $|\nabla u| \in L_{1}^{\infty}$. The following lemma shows that, in this sense, Corollary 1 improves [12, Theorem 7], according to which the same conclusion holds if the linearly independent solutions $f_{1}, f_{2} \in \mathcal{B}$ satisfy $\inf _{z \in \mathbb{D}}\left(\left|f_{1}(z)\right|+\left|f_{2}(z)\right|\right)>0$.

\section{Lemma 6 The following assertions hold.}

(i) If $f_{1}, f_{2} \in \mathcal{B}$ are linearly independent solutions of (1) for $A \in \operatorname{Hol}(\mathbb{D})$, and $\inf _{z \in \mathbb{D}}\left(\left|f_{1}(z)\right|+\left|f_{2}(z)\right|\right)>0$, then $|\nabla u| \in L_{1}^{\infty}$ for $u=-\log \left(f_{1} / f_{2}\right)^{\#}$.

(ii) There exists $A \in H_{2}^{\infty}$ such that (1) admits linearly independent solutions $f_{1}, f_{2}$ such that $\inf _{z \in \mathbb{D}}\left(\left|f_{1}(z)\right|+\left|f_{2}(z)\right|\right)=0$ but $|\nabla u| \in L_{1}^{\infty}$.

(iii) There exists $A \in \operatorname{Hol}(\mathbb{D})$ such that (1) admits linearly independent solutions $f_{1}, f_{2}$ with $f_{1} / f_{2}$ bounded (and hence normal) but $|\nabla u| \notin L_{1}^{\infty}$. 
Proof (i) Since $f_{1}, f_{2} \in \mathcal{B}$ satisfy $\inf _{z \in \mathbb{D}}\left(\left|f_{1}(z)\right|+\left|f_{2}(z)\right|\right)>0$, we deduce

$$
\begin{aligned}
|\nabla u(z)| & =2|\partial u(z)|=2 \frac{\left|f_{1}^{\prime}(z) \overline{f_{1}(z)}+f_{2}^{\prime}(z) \overline{f_{2}(z)}\right|}{\left|f_{1}(z)\right|^{2}+\left|f_{2}(z)\right|^{2}} \\
& \leq \frac{2 \max \left\{\left\|f_{1}\right\|_{\mathcal{B}},\left\|f_{2}\right\|_{\mathcal{B}}\right\}}{1-|z|^{2}} \frac{\left|f_{1}(z)\right|+\left|f_{2}(z)\right|}{\left|f_{1}(z)\right|^{2}+\left|f_{2}(z)\right|^{2}} \lesssim \frac{1}{1-|z|^{2}}, \quad z \in \mathbb{D} .
\end{aligned}
$$

(ii) Consider the analytic and univalent function $h(z)=-\log (1-z), z \in \mathbb{D}$. Define $A=S_{h} / 2$, where $S_{h}$ is the Schwarzian derivative of $h$. Then, $A(z)=4^{-1}(1-z)^{-2}, z \in \mathbb{D}$, and clearly $A \in H_{2}^{\infty}$. It is well-known that (1) admits two linearly independent solutions $f_{1}, f_{2}$ such that $h=f_{1} / f_{2}$. In this case

$$
\frac{\left|W\left(f_{1}, f_{2}\right)\right|}{\left|f_{1}(z)\right|^{2}+\left|f_{2}(z)\right|^{2}}=h^{\#}(z)=\frac{1}{|1-z|\left(1+|\log (1-z)|^{2}\right)}, \quad z \in \mathbb{D},
$$

is unbounded in $\mathbb{D}$, while $|\nabla u| \in L_{1}^{\infty}$ by Corollary 1 ( $h$ is normal as it is univalent).

Part (iii) follows by the proof of Theorem 1(ii). An application of Corollary 1 reveals that $|\nabla u| \notin L_{1}^{\infty}$.

It is a natural question to ask how $|\nabla u| \in L_{1}^{\infty}$ compares to Theorem 15? On one hand, Lemma 6(ii) serves as an example where $|\nabla u| \in L_{1}^{\infty}$ but (12) fails for any pairwise disjoint pseudo-hyperbolic discs (consider the positive real axis). On the other hand, Example 1(ii) in Sect. 4 implies that there are cases in which (12) is satisfied but $|\nabla u| \notin L_{1}^{\infty}\left(f_{1} / f_{2}\right.$ is not normal). In both of these examples, the coefficient function satisfies $A \in H_{2}^{\infty}$.

Proof (of Corollary 2) The assertions (i) and (ii) are equivalent by Theorem 11 . Note that (i) implies (iii) by Lemma 5, while (iii) implies (i), and also (ii), by Theorem 5(ii). Finally, (ii) is equivalent to (iv) according to Theorem 5(i).

The arguments in this section are build on the representation (13) for the coefficient $A$. Derivatives of the coefficient can be controlled by expressions of similar type. For example, by differentiating (1) we obtain $f^{\prime \prime \prime}+A^{\prime} f+A f^{\prime}=0$, and

$$
\begin{aligned}
|A| & =\frac{\left|f_{1}^{\prime}\right|+\left|f_{2}^{\prime}\right|}{\left|f_{1}^{\prime}\right|+\left|f_{2}^{\prime}\right|}|A|=\frac{\left|f_{1}^{\prime \prime \prime}+A^{\prime} f_{1}\right|+\left|f_{2}^{\prime \prime \prime}+A^{\prime} f_{2}\right|}{\left|f_{1}^{\prime}\right|+\left|f_{2}^{\prime}\right|} \\
& \geq\left|A^{\prime}\right| \frac{\left|f_{1}\right|+\left|f_{2}\right|}{\left|f_{1}^{\prime}\right|+\left|f_{2}^{\prime}\right|}-\frac{\left|f_{1}^{\prime \prime \prime}\right|+\left|f_{2}^{\prime \prime \prime}\right|}{\left|f_{1}^{\prime}\right|+\left|f_{2}^{\prime}\right|} .
\end{aligned}
$$

Therefore, by applying (13),

$$
\left|A^{\prime}\right| \leq \frac{\left|f_{1}^{\prime}\right|+\left|f_{2}^{\prime}\right|}{\left|f_{1}\right|+\left|f_{2}\right|}\left(|A|+\frac{\left|f_{1}^{\prime \prime \prime}\right|+\left|f_{2}^{\prime \prime \prime}\right|}{\left|f_{1}^{\prime}\right|+\left|f_{2}^{\prime}\right|}\right)=\frac{\left|f_{1}^{\prime}\right|+\left|f_{2}^{\prime}\right|}{\left|f_{1}\right|+\left|f_{2}\right|} \cdot \frac{\left|f_{1}^{\prime \prime}\right|+\left|f_{2}^{\prime \prime}\right|}{\left|f_{1}\right|+\left|f_{2}\right|}+\frac{\left|f_{1}^{\prime \prime \prime}\right|+\left|f_{2}^{\prime \prime \prime}\right|}{\left|f_{1}\right|+\left|f_{2}\right|} .
$$

\section{Proof of Theorem 12}

It is natural to require that solution with prescribed fixed points is bounded in $\mathbb{D}$. Under this requirement, Theorem 12 is best possible. This is a consequence of the following auxiliary result.

Lemma 7 The following assertions hold. 
(i) The identity function is the only one in $\left\{f \in H^{\infty}:\|f\|_{H^{\infty}} \leq 1\right\}$ which has more than one fixed point.

(ii) The identity function is the only one in $\mathcal{N}$ which has more fixed points than the Blaschke condition allows.

The proof of the lemma is straight-forward and hence omitted.

Proof (of Theorem 12) Let $B=B_{\left\{z_{n}\right\}}$ be the Blaschke product with zeros $\left\{z_{n}\right\}$. Let $0<\varepsilon<1$, and define $f_{1}(z)=z+\varepsilon z^{3} B(z), z \in \mathbb{D}$. The fixed points of $f_{1}$ are precisely $\{0\} \cup\left\{z_{n}\right\}$. By the Schwarz lemma $\left|z^{3} B(z)\right| \leq|z|$ for $z \in \mathbb{D}$, and therefore $(1-\varepsilon)|z| \leq\left|f_{1}(z)\right| \leq(1+\varepsilon)|z|$ for any $z \in \mathbb{D}$.

Since $f_{1}$ has only one zero in $\mathbb{D}$ and $f_{1}^{\prime \prime}(0)=0$, we deduce $A=-f_{1}^{\prime \prime} / f_{1} \in \operatorname{Hol}(\mathbb{D})$. If $0<\delta<1$, then

$$
\sup _{\delta<|z|<1}|A(z)| \leq \frac{\varepsilon}{(1-\varepsilon) \delta} \sup _{\delta<|z|<1}\left(\left|B^{\prime \prime}(z)\right|+6\left|B^{\prime}(z)\right|+6|B(z)|\right),
$$

and consequently, $|A(z)|^{2}\left(1-|z|^{2}\right)^{3} d m(z)$ is a Carleson measure. If $f_{2}$ is defined by (19) for fixed $\alpha \in \mathbb{D} \backslash\{0\}$, then $f_{2} \in H^{\infty}$ is a solution of (1) and is linearly independent to $f_{1}$. Consequently, all solutions of (1) are bounded.

\section{Proofs of Theorems 13 and 14}

Proof (of Theorem 13) Let $\Lambda \subset \mathbb{D} \backslash\{0\}$ be a uniformly separated sequence. Then, the corresponding Blaschke product $B=B_{\Lambda}$ satisfies (20).

Let $h \in H^{\infty}$ be a function which satisfies $h\left(z_{n}\right)=\log z_{n}$ for $z_{n} \in \Lambda$. The existence of such $h$ is guaranteed by Carleson's interpolation theorem [2, Theorem 3]. Let $\left\{C_{n}\right\}$ be the sequence of real numbers defined as follows: Whenever $z_{n} \in \Lambda$ is prescribed to be an attractive fixed point define $C_{n}=1 / 2$, if neutral choose $C_{n}=1$, while otherwise take $C_{n}=2$. By (20), we obtain

$$
\sup _{z_{n} \in \Lambda}\left|\frac{1}{B^{\prime}\left(z_{n}\right)}\left(\frac{C_{n}}{z_{n}}-h^{\prime}\left(z_{n}\right)\right)\right| \leq \sup _{z_{n} \in \Lambda} \frac{1-\left|z_{n}\right|^{2}}{\delta}\left(\frac{2}{\inf _{n}\left|z_{n}\right|}+\left|h^{\prime}\left(z_{n}\right)\right|\right)<\infty,
$$

and hence $\left\{w_{n}\right\}=\left\{\left(C_{n} / z_{n}-h^{\prime}\left(z_{n}\right)\right) / B^{\prime}\left(z_{n}\right)\right\}$ is a bounded sequence. The aforementioned Carleson's result guarantees that there exists $g \in H^{\infty}$ with $g\left(z_{n}\right)=w_{n}$ for $z_{n} \in \Lambda$. Define $f_{1}=\exp (h+B g)$, and note that $f_{1}$ is not only in $H^{\infty}$ but also is uniformly bounded away from zero. Moreover,

$$
f_{1}\left(z_{n}\right)=z_{n}, \quad f_{1}^{\prime}\left(z_{n}\right)=z_{n}\left(h^{\prime}\left(z_{n}\right)+B^{\prime}\left(z_{n}\right) g\left(z_{n}\right)\right)=C_{n}, \quad z_{n} \in \Lambda .
$$

The points $z_{n} \in \Lambda$ are fixed points of the prescribed type. The coefficient $A=-f_{1}^{\prime \prime} / f_{1} \in$ $\operatorname{Hol}(\mathbb{D})$ satisfies $|A| \lesssim\left|f_{1}^{\prime \prime}\right|$ in $\mathbb{D}$, and therefore $|A(z)|^{2}\left(1-|z|^{2}\right)^{3} d m(z)$ is a Carleson measure. The fact that all solutions of (1) are bounded follows as in the proof of Theorem 12.

Note that the solution $f_{1}$ in Theorem 13, which has prescribed fixed points of pregiven type, may have fixed points which do not belong to $\Lambda$.

Remark 3 If $A \in \operatorname{Hol}(\mathbb{D})$ and $z_{0} \in \mathbb{D}$, then (1) admits a unique solution $f$ such that the initial conditions $f\left(z_{0}\right)=\alpha \in \mathbb{C}$ and $f^{\prime}\left(z_{0}\right)=\beta \in \mathbb{C}$ are satisfied. Therefore fixed 
points of solutions of (1) are not always distinct from zeros or critical points. In the proof of Theorem $13,\left\{C_{n}\right\} \subset \mathbb{C}$ can be any sequence with the property $\sup _{n}\left|C_{n}\right|\left(1-\left|z_{n}\right|^{2}\right)<\infty$. If we take $C_{n}=0$ for all $n$, then every point $z_{n} \in \Lambda$ is not only a fixed point but also a critical point of the solution $f_{1}$.

Proof (of Theorem 14) Let $\Lambda \in \operatorname{Int} \mathcal{N}$ be the sequence of non-zero points, and let $B=B_{\Lambda}$ be the corresponding Blaschke product. Since $\Lambda \in \operatorname{Int} \mathcal{N}$, [18, Theorem 1.2] implies that there exists $h_{1} \in \mathrm{Har}^{+}(\mathbb{D})$ such that (31) holds.

Let $h \in \mathcal{N}$ be a function which satisfies $h\left(z_{n}\right)=\log z_{n}$ for $z_{n} \in \Lambda$. Since $\Lambda$ is Nevanlinna interpolating, the existence of such function $h$ is guaranteed by [18, Theorem 1.2]. Let $\left\{C_{n}\right\}$ be the sequence of real numbers defined as in the proof of Theorem 13. As $h \in \mathcal{N}$, (29) implies that there exists a constant $0<C<\infty$ and $h_{2} \in \operatorname{Har}^{+}(\mathbb{D})$ such that

$$
\left|\frac{1}{B^{\prime}\left(z_{n}\right)}\left(\frac{C_{n}}{z_{n}}-h^{\prime}\left(z_{n}\right)\right)\right| \leq \frac{1-\left|z_{n}\right|^{2}}{e^{-h_{1}\left(z_{n}\right)}}\left(\frac{2}{\inf _{n}\left|z_{n}\right|}+\frac{e^{C} e^{h_{2}\left(z_{n}\right)}}{1-\left|z_{n}\right|^{2}}\right), \quad z_{n} \in \Lambda .
$$

Since $\left\{w_{n}\right\}=\left\{\left(C_{n} / z_{n}-h^{\prime}\left(z_{n}\right)\right) / B^{\prime}\left(z_{n}\right)\right\} \in \mathcal{N} \mid \Lambda$ by [18, Theorem 1.2], there exists $g \in \mathcal{N}$ with $g\left(z_{n}\right)=w_{n}$ for $z_{n} \in \Lambda$. Define $f=\exp (h+B g)$, and note that

$$
f\left(z_{n}\right)=z_{n}, \quad f^{\prime}\left(z_{n}\right)=z_{n}\left(h^{\prime}\left(z_{n}\right)+B^{\prime}\left(z_{n}\right) g\left(z_{n}\right)\right)=C_{n}, \quad z_{n} \in \Lambda .
$$

The points $z_{n} \in \Lambda$ are fixed points of the prescribed type. Finally, the coefficient

$$
A=-f^{\prime \prime} / f=-\left((h+B g)^{\prime}\right)^{2}-(h+B g)^{\prime \prime} \in \operatorname{Hol}(\mathbb{D})
$$

satisfies $|A(z)|\left(1-|z|^{2}\right)^{2} \leq e^{H(z)}, z \in \mathbb{D}$ and $H \in \operatorname{Har}^{+}(\mathbb{D})$, by (29).

Acknowledgements The author thanks the anonymous referee for valuable suggestions and Artur Nicolau for helpful conversations, especially in relation to Nevanlinna interpolating sequences. The author gratefully acknowledges the hospitality of Departament de Matemàtiques, Universitat Autònoma de Barcelona.

Funding Open access funding provided by University of Eastern Finland (UEF) including Kuopio University Hospital.

Open Access This article is licensed under a Creative Commons Attribution 4.0 International License, which permits use, sharing, adaptation, distribution and reproduction in any medium or format, as long as you give appropriate credit to the original author(s) and the source, provide a link to the Creative Commons licence, and indicate if changes were made. The images or other third party material in this article are included in the article's Creative Commons licence, unless indicated otherwise in a credit line to the material. If material is not included in the article's Creative Commons licence and your intended use is not permitted by statutory regulation or exceeds the permitted use, you will need to obtain permission directly from the copyright holder. To view a copy of this licence, visit http://creativecommons.org/licenses/by/4.0/.

\section{References}

1. Arbeláez, H., Mejía, D.: On spherical invariance. Rev. Colombiana Mat. 45(1), 97-112 (2011)

2. Carleson, L.: Interpolations by bounded analytic functions and the corona problem. Ann. Math. 2(76), 547-559 (1962)

3. Chuaqui, M., Gröhn, J., Heittokangas, J., Rättyä, J.: Zero separation results for solutions of second order linear differential equations. Adv. Math. 245, 382-422 (2013)

4. Cima, J.A., Colwell, P.: Blaschke quotients and normality. Proc. Am. Math. Soc. 19, 796-798 (1968)

5. Duren, P., Schuster, A.: Bergman Spaces, Mathematical Surveys and Monographs, 100. American Mathematical Society, Providence (2004)

6. Earl, J.P.: On the interpolation of bounded sequences by bounded functions. J. Lond. Math. Soc. (2) 2 , 544-548 (1970) 
7. Fournier, R., Kraus, D., Roth, O.: A Schwarz lemma for locally univalent meromorphic functions. Proc. Am. Math. Soc. 148, 3859-3870 (2020)

8. Garnett, J.: Bounded Analytic Functions, Revised First Edition. Graduate Texts in Mathematics, vol. 236. Springer, New York (2007)

9. Gröhn, J.: New applications of Picard's successive approximations. Bull. Sci. Math. 135(5), 475-487 (2011)

10. Gröhn, J.: On non-normal solutions of linear differential equations. Proc. Am. Math. Soc. 145(3), 12091220 (2017)

11. Gröhn, J.: Solutions of complex differential equation having pre-given zeros in the unit disc. Constr. Approx. 49(2), 295-306 (2019)

12. Gröhn, J.: Slowly growing solutions of ODEs revisited. Ann. Acad. Sci. Fenn. Math. 43, 617-629 (2018)

13. Gröhn, J., Heittokangas, J.: New findings on Bank-Sauer approach in oscillation theory. Constr. Approx. 35(3), 345-361 (2012)

14. Gröhn, J., Huusko, J.-M., Rättyä, J.: Linear differential equations with slowly growing solutions. Trans. Am. Math. Soc. 370(10), 7201-7227 (2018)

15. Gröhn, J., Nicolau, A.: Uniform separation, hyperbolic geodesics and zero distribution of solutions of linear differential equations. Bull. Lond. Math. Soc. 49(3), 380-390 (2017)

16. Gröhn, J., Rättyä, J.: On oscillation of solutions of linear differential equations. J. Geom. Anal. 27(1), 868-885 (2017)

17. Gröhn, J., Nicolau, A., Rättyä, J.: Mean growth and geometric zero distribution of solutions of linear differential equations. J. Anal. Math. 134(2), 747-768 (2018)

18. Hartmann, A., Massaneda, X., Nicolau, A., Thomas, P.: Interpolation in the Nevanlinna and Smirnov classes and harmonic majorants. J. Funct. Anal. 217(1), 1-37 (2004)

19. Hartmann, A., Massaneda, X., Nicolau, A.: Finitely generated ideals in the Nevanlinna class. Israel J. Math. 231(1), 139-179 (2019)

20. Heittokangas, J.: On complex differential equations in the unit disc. Dissertation, University of Joensuu, Joensuu, 2000. Ann. Acad. Sci. Fenn. Math. Diss. No. 122, p 54 (2000)

21. Heittokangas, J.: On interpolating Blaschke products and Blaschke-oscillatory equations. Constr. Approx. 34(1), 1-21 (2011)

22. Heittokangas, J.: A Survey on Blaschke-Oscillatory Differential Equations, with Updates, Blaschke Products and Their Applications, Fields Institute Communications, vol. 65, pp. 43-98. Springer, New York (2013)

23. Heittokangas, J., Korhonen, R., Rättyä, J.: Linear differential equations with solutions in the Dirichlet type subspace of the Hardy space. Nagoya Math. J. 187, 91-113 (2007)

24. Huusko, J.-M., Korhonen, T., Reijonen, A.: Linear differential equations with solutions in the growth space $H_{\omega}^{\infty}$. Ann. Acad. Sci. Fenn. Math. 41(1), 399-416 (2016)

25. Kennedy, P.B.: On the derivative of a function of bounded characteristic. Q. J. Math. Oxford Ser. (2) 15, 337-341 (1964)

26. Kerr-Lawson, A.: Some lemmas on interpolating Blaschke products and a correction. Can. J. Math. 21, 531-534 (1969)

27. Laine, I.: Nevanlinna Theory and Complex Differential Equations. Walter de Gruyter, Berlin (1993)

28. Lappan, P.: A non-normal locally uniformly univalent function. Bull. Lond. Math. Soc. 5, 291-294 (1973)

29. Lappan, P.: On the normality of derivatives of functions. Math. Ann. 238(2), 141-146 (1978)

30. Lehto, O., Virtanen, K.I.: Boundary behaviour and normal meromorphic functions. Acta Math. 97, 47-65 (1957)

31. Liouville, J.: Sur l'équation aux différences partielles $\frac{d^{2} \log \lambda}{d u d v} \pm \frac{\lambda}{2 a^{2}}=0$. J. Math. 16, 71-72 (1853)

32. Massaneda, X., Nicolau, A., Thomas, P.: The Corona property in Nevanlinna quotient algebras and interpolating sequences. J. Funct. Anal. 276(8), 2636-2661 (2019)

33. Mortini, R.: Zur Idealstruktur von Unterringen der Nevanlinna-Klasse $N$, Travaux mathématiques, I, 81-91, Sém. Math. Luxembourg, Centre Univ. Luxembourg, Luxembourg (1989)

34. Nehari, Z.: The Schwarzian derivative and schlicht functions. Bull. Am. Math. Soc. 55, 545-551 (1949)

35. Nicolau, A.: Finite products of interpolating Blaschke products. J. Lond. Math. Soc. (2) 50(3), 520-531 (1994)

36. Nikolskii, N.K.: Treatise on the Shift Operator. Springer, New York (1985)

37. Øyma, K.: Interpolation in $H^{p}$-spaces. Proc. Am. Math. Soc. 76(1), 81-88 (1979)

38. Pavićević, Ž.: The Carleson measure and meromorphic functions of uniformly bounded characteristic. Ann. Acad. Sci. Fenn. Ser. A I Math. 16(2), 249-254 (1991)

39. Peláez, J.Á., Rättyä, J.: Weighted Bergman spaces induced by rapidly increasing weights. Mem. Am. Math. Soc. 227(1066), vi+124 (2014) 
40. Pommerenke, Ch.: On the mean growth of the solutions of complex linear differential equations in the disk. Complex Var. Theory Appl. 1(1), 23-38 (1982/83)

41. Rosenblum, M., Rovnyak, J.: Topics in Hardy Classes and Univalent Functions. Birkhäuser Verlag, Basel (1994)

42. Rättyä, J.: On some complex function spaces and classes. Ann. Acad. Sci. Fenn. Math. Diss. No. 124, 73 (2001)

43. Schwarz, B.: Complex nonoscillation theorems and criteria of univalence. Trans. Am. Math. Soc. 80, 159-186 (1955)

44. Steinmetz, N.: Normal families and linear differential equations. J. Anal. Math. 117, 129-132 (2012)

45. Tolokonnikov, V.A.: Blaschke products satisfying the Carleson-Newman conditions and ideals of the algebra $H^{\infty}$. J. Soviet Math. 42(2), 1603-1610 (1988)

46. Treil, S., Wick, B.: The matrix-valued $H^{p}$ corona problem in the disk and polydisk. J. Funct. Anal. 226(1), 138-172 (2005)

47. Yamashita, S.: Functions of uniformly bounded characteristic. Ann. Acad. Sci. Fenn. Ser. A I Math. 7(2), 349-367 (1982)

48. Yamashita, S.: Estimates of spherical derivative of meromorphic functions. Hokkaido Math. J. 31(1), 151-186 (2002)

Publisher's Note Springer Nature remains neutral with regard to jurisdictional claims in published maps and institutional affiliations. 